\title{
Soluble Brain Homogenates from Diverse Human and Mouse Sources Preferentially Seed Diffuse A $\beta$ Plaque Pathology When Injected into Newborn Mouse Hosts
}

\section{Brenda Moore ( $\sim$ brendar@ufl.edu )}

University of Florida https://orcid.org/0000-0002-9685-6561

\section{Yona Levites}

University of Florida Department of Neuroscience

\section{Guilian Xu}

University of Florida Department of Neuroscience

\section{Hailey Hampton}

University of Florida Department of Neuroscience

\section{Munir F Adamo}

University of Florida Department of Neuroscience

Cara L. Croft

The Francis Crick Institute

\section{Hunter S Futch}

University of Florida Department of Neuroscience

\section{Corey Moran}

University of Florida Department of Neuroscience

\section{Susan Fromholt}

University of Florida Department of Neuroscience

Christopher Janus

University of Florida Department of Neuroscience

\section{Stefan Prokop}

University of Florida Department of Neuroscience

\section{Dennis Dickson}

University of Florida Department of Neuroscience Jada Lewis

University of Florida Department of Neuroscience

\section{Benoit I. Giasson}

University of Florida Department of Neuroscience

\section{Todd E Golde}

University of Florida Department of Neuroscience 


\section{David R. Borchelt}

University of Florida Department of Neuroscience

\section{Research Article}

Keywords: amyloid- $\beta$, tau, a-synuclein, seeding, Alzheimer's disease, Lewy body dementia

Posted Date: November 10th, 2021

DOI: https://doi.org/10.21203/rs.3.rs-1040944/v1

License: (c) (1) This work is licensed under a Creative Commons Attribution 4.0 International License. Read Full License 


\section{Abstract \\ Background}

Seeding of pathology related to Alzheimer's disease (AD) and Lewy body disease (LBD) by the injection of tissue homogenates, purified proteins, or recombinant proteins into model systems has revealed prionlike seeding of the protein aggregates that define these disorders. Most commonly these homogenates are injected into adult mice stereotaxically. Injection of brain lysates into newborn mice represents an alternative approach of delivering seeds that could be used to direct the evolution of amyloid- $\beta$ (A $\beta)$ pathology co-mixed with either tau or a-synuclein (aSyn) pathology in vulnerable mouse models.

\section{Methods}

Homogenates of human pre-frontal cortex were prepared and injected into the lateral ventricles of newborn (P0) mice expressing a mutant humanized amyloid precursor protein (APP), human P301L tau, human wild type aSyn, or combinations thereof. The injected brain homogenates were prepared from $A D$ and $A D / L B D$ cases displaying variable degrees of $A \beta$ pathology and co-existing tau and aSyn deposits. Behavioral assessments of APP transgenic mice injected with AD brain lysates were conducted.

\section{Results}

We observed that the lysates from the brains of individuals with $A D(A \beta+$, tau+), $A D / L B D(A \beta+$, tau+, aSyn+), or Pathological Aging ( $A \beta+$, tau-, aSyn-) efficiently seeded diffuse $A \beta$ deposits, composed primarily of $A \beta 42$ peptides, in our transgenic host animals. Moderate seeding of cerebral amyloid angiopathy (CAA) was also observed. No animal of any genotype developed discernable tau or aSyn pathology. Fear conditioning, cognitive, outcome was not significantly altered in APP transgenic animals injected with AD brain lysates compared to nontransgenic controls.

\section{Conclusions}

These findings demonstrate that diffuse $A \beta$ pathology, which is a common feature of $A D, A D / L B D$, and PA brains, can be easily induced by injecting newborn APP mice with crude brain homogenates. Seeding of tau or aSyn comorbidities was disappointingly inefficient in the models we used, indicating additional methodological refinement will be needed to efficiently seed AD or AD/LBD mixed pathologies by injecting newborn mice.

\section{Background}

The amyloid cascade hypothesis posits that deposition of the $A \beta$ peptide is an initiating factor in Alzheimer's disease (AD) with downstream effects of increased inflammatory response, altered neuronal 
homeostasis, altered tau phosphorylation leading to tangle formation, neuronal dysfunction, and cognitive decline $(1,2)$. A $\beta$ containing seeds, derived from human $A D$ and Pathological Aging (PA) brain lysates, have been shown to accelerate the time-course and severity of $A \beta$ deposition in amyloid precursor protein (APP) transgenic models as well as impact the morphology of the seeded deposits (for review see (3)). The literature contains numerous studies in which different types of seeding preparations have been injected in different APP mouse models [reviewed in (3)]. Seeding preparations can consist of human brain homogenates, APP transgenic mouse brain homogenates, enriched preparations derived from such homogenates and synthetic $A \beta$ fibrils (3). In most of these published studies, seeds are injected into adult APP mice that are a few months away from developing pathology on their own and in such cases the inherent pathological tendencies of the APP host model can contribute to the pathological outcome.

Recent studies have begun to use seeding approaches to create models of mixed AD or Lewy body disease (LBD) pathology. Partially purified tau aggregates from $A D$ brains were found to seed enhanced neuritic tau pathology in the 5xFAD mouse model and the NL-G-F/human MAPT double knock-in model (4). In Parkinson's disease and LBD, the presence of $A \beta$ plaques is associated with cortical aSyn aggregation and spreading $(5,6)$. A number of recent studies suggest a possible synergistic relationship between aSyn and $A \beta(7)$. $A \beta$ deposits accelerated aSyn pathogenesis and spread throughout the brain of 5xFAD mice (8), whereas reducing aSyn expression in transgenic APP mice rescued and reversed the amyloid pathology and neurodegenerative phenotype (9). One of the goals of our study was to explore the potential to use seeding as a means to generate novel mouse models of mixed AD and LBD pathology.

A subset of cognitively normal aged individuals exhibit substantial A $\beta$ pathology at autopsy (10). These individuals, which we use the designation of Pathologic Aging (PA), may represent prodromal, presymptomatic $A D$ (11-14). The amyloid pathology of PA brains has typically been described as diffuse in nature whereas $A D$ brains contain both diffuse and compact/neuritic deposits of $A \beta$ (14-16). Cognitively normal individuals with PA generally have very low levels of tau pathology (14). Rigorous biochemical examination of $A \beta$ from the pre-frontal cortex of PA and AD brain revealed extensive overlap in A $\beta$ levels, peptide profiles, solubility, and SDS-stable oligomeric assemblies (13). Interestingly, brain homogenates from a human PA case were shown to seed diffuse A $\beta$ deposition in APP transgenic mice, albeit at somewhat less severe levels (17). Thus, PA brains provide a source of $A \beta$ seeds that are less contaminated with tau seeds and therefore provide a means to examine the potential for seeded $A \beta$ pathology to trigger tau pathology in APP/tau mice.

Our approach for this study was to inject homogenates prepared from $A D, P A$, and $A D / L B D$ donors into newborn APP, APP/tau, or APP/aSyn host mice. In prior studies, we had established that injection of recombinant adeno-associated virus into newborn mice was an effective approach to obtaining widespread dispersion of injected virus $(18,19)$. We therefore hypothesized that misfolded seeds of $A \beta$, tau, or aSyn might similarly be more widely dispersed to more effectively seed pathology. In fact, we observed efficient seeding of aSyn pathology in newborn mice, expressing wild-type human aSyn (M20 
line), by injecting brain lysates from patients with multisystem atrophy (20). Here we performed brain lysate injection studies at PO in the lateral ventricles of host transgenic mice that co-express humanized mutant APP with either mutant human tau-P301L or wild-type human aSyn (M20 line). Importantly, the recipient transgenic mice used in the current studies develop pathology at late ages or not at all; enabling sensitive evaluation of potential seeding. Seeding of APP, APP/tau, or APP/aSyn mice with these brain homogenates failed to produce tau or aSyn pathology but efficiently induced diffuse $A \beta$ pathology. Notably, homogenates from human brain with high levels of cerebral amyloid angiopathy (CAA) also seeded modest CAA. We compared the data from human lysates to lysates prepared from transgenic mouse donors finding again that diffuse $A \beta$ deposits were preferentially seeded. These findings demonstrate that diffuse $A \beta$ pathology can readily be induced by injecting newborn APP, APP/tau, or APP/aSyn mice with homogenates from diverse pathological specimens. Though the human homogenates used contained coexisting aSyn and tau pathologies, we were not able to directly induce LBD or tau pathology in recipient models tested here. Moreover, the induced diffuse $A \beta$ pathology failed to secondarily induce LBD or tau pathology in mice that were bigenic for APP/tau or APP/aSyn. APP mice seeded with $A D$ brain lysates, exhibiting diffuse $A \beta$ pathology, also showed no significant impairments in a contextual fear paradigm of cognitive performance. These findings are consistent with the idea that diffuse $A \beta$ pathology may be less pathogenic than cored-neuritic deposits (21).

\section{Methods}

\section{Description of AD, PA and NDC cases}

For this study we utilized a subset of the human brain samples that our group previously analyzed (13). Tissue sections and frozen pre-frontal cortex $(A D=2, P A=4$, and $N D C=2)$ were obtained from the Mayo Clinic Brain Bank with informed consent, in accordance with the Mayo Clinic institutional review board, using previously described acquisition and diagnostic analyses $(14,22,23)$. Samples were initially cryopulverized to allow for multiple studies. We previously analyzed the $A \beta$ peptide profile, quantity, and solubility in these brain samples (13). In this study, we analyzed two brains from AD patients (both aged 84 ), four PA brains from subjects (age range $=78$ to 83 ) without clinical evidence of dementia, and two brains with rare or no $A D$ lesions from elderly individuals without clinical evidence of a neurological illness (ages 75 and 78). In addition, human brain samples were also obtained from the University of Florida Neuromedicine Human Brain Tissue Bank following institutional regulations and previously described classification (24-26). We analyzed tissue sections and frozen pre-frontal cortex from two AD patients (ages 82 and 86), two cases with AD/LBD (ages 81 and 83), and two control individuals without pathology (ages 52 and 82 ). Table 1 summarizes the cases studied in this report.

\section{Transgenic mice}

The transgenic mice used in this study have been described previously and are summarized in Table 2 . The PrP.APPsi mice express human/mouse APPswe/ind using the MoPrP.Xho vector, which was coinjected with a vector to express eGFP in skin (first described in (27)). The iTau-P301L mice express 
human 4R0N tau-P301L using a tet-regulated vector that includes a minimal CMV promoter (28). Tau levels were assessed by Western blotting. PBS lysates of 2.5-6 month old P301L mice were heated at $95^{\circ} \mathrm{C}$ for five minutes in the presence of denaturing SDS sample buffer, were separated on a $4-12 \%$ BisTris gel (Bio-Rad, Hercules, CA) in 3-(N-morpholino)propanesulfonic acid (MOPS) running buffer (Bio-Rad) and transferred onto PVDF membrane. The membrane was blocked in casein blocking buffer and incubated overnight at $4^{\circ} \mathrm{C}$ with primary antibodies to tau, Tau-13 (anti-human tau2-18; Covance) and 3026 (rabbit polyclonal antibody raised against full length ON3R human tau and also reacts with ON4R human tau $(29,30)$ ), and anti- $\beta$-actin (Sigma-Aldrich, St. Louis, MO) before incubation with secondary antibody, fluorophore conjugated AlexaFluor 680 anti-mouse IgG (Thermo Fisher Scientific, Waltham, MA). Protein bands were detected and quantified using the multiplex Odyssey Infrared Imaging system (Bio-Rad, Hercules, CA).

The M20 mice express wild type aSyn under the control of the prion promoter and do not develop aSyn pathology (31). PrP.HuAß/PS1 mice express human APPswe and human PS1dE9. PrP.MoAß/PS1 mice express mouse APPswe mutation and human PS1dE9, and tet.MoA $\beta$ mice expresses inducible mouse APPswe (27).

These mice were bred in-house. PrP.APPsi and M20 were maintained on hybrid C57BL6/C3H backgrounds, following a breeding scheme in which transgene positive males were breed to $\mathrm{F} 1 \mathrm{~B} 6 / \mathrm{C} 3$ mice. The iTau-P301L were maintained on a background of FVB mice. APPsi/Tau-P301L and APPsi/aSyn mice were generated by intercrossing mice that where hemizygous for the respective transgenes.

PrP.APPsi mice were genotyped by visualizing GFP expression, which is possible because these mice were co-injected with a transgene that expresses GFP in the skin integrated next to APP transgene. PrP.APPsi/iTau-P301L mice were genotpyed for APP by illumination and visulation with special filter goggles (BLS Ltd, Budapest, Hungary) and for tau by PCR of tail DNA. iTau-P301L and M20 mice were genotyped by PCR of tail DNA. PrP.APPsi destined for behavioral studies were backcrossed one generation onto C57BL6. All animals were housed 1-5 per cage with unlimited access to food and water with a 14-hour light and 10-hour dark cycle. All experiments involving mice were approved by the University of Florida Institutional Animal Care and Use Committee (IACUC) and conducted in accordance with NIH guidelines.

\section{Human brain lysates}

Human and mouse brain lysates were prepared as previously described (32). Briefly, frozen tissue was homogenized at $10 \%(\mathrm{w} / \mathrm{v})$ in sterile PBS, subjected to vortex and sonication $(3 \times 5 \mathrm{sec})$ and then centrifuged $3000 \mathrm{xg}$ for $5 \mathrm{~min}$ at $4^{\circ} \mathrm{C}$. Lysates were immediately aliquoted and stored at $-80^{\circ} \mathrm{C}$ until needed. As previously described (13), the $A \beta$ levels and solubility in the lysates was assessed by Western blotting. Briefly, lysates were heated at $50^{\circ} \mathrm{C}$ for three minutes in the presence of denaturing sample buffer, were separated on a 4-12\% Bis-Tris gel (Bio-Rad, Hercules, CA) in 1X2-(Nmorpholino)ethanesulfonic acid (MES) running buffer (Bio-Rad) and transferred onto nitrocellulose membrane (Bio-Rad, Hercules, CA). The membrane was boiled in PBS for $5 \mathrm{~min}$, blocked in Starting Block 
(Thermo Fisher Scientific (formally Thermo Scientific), Waltham, MA) and incubated overnight at $4^{\circ} \mathrm{C}$ with primary antibody Ab5 (human AB1-16 specific; T.E. Golde) before incubation with the secondary antibody, fluorophore conjugated Alexa Fluor 680 anti-mouse IgG (Thermo Fisher Scientific, Waltham, MA). Protein bands were detected using the multiplex Odyssey Infrared Imaging system (Bio-Rad, Hercules, CA).

\section{Brain seeding with neonatal cerebral ventricle injections}

Injections of human brain lysates and lysates from a 27mo PrP.APPsi mouse, a 25mo PrP.HuAß/PS1 mouse, a 24mo Tet.MoA $\beta$ mouse, and a 24mo PrP.MoA $\beta / P S 1$ mouse were performed as described previously with recombinant adeno-associated virus (33). Briefly, P0 pups were cryoanesthisized and $2 \mathrm{uL}$ of human or mouse brain lysates were bilaterally injected into the cerebral ventricle using a $10 \mathrm{uL}$ Hamilton syringe with a 30 -inch needle (Hamilton Company, Reno, NV). Pups were placed on a heating pad for recovery and returned to their home cage. Cohorts of mice were euthanized at $6,9,12$, and 18 months for analysis.

\section{Mouse brain tissue collection}

Mice were anesthetized with isoflurane and perfused transcardially with $20 \mathrm{~mL}$ of cold PBS. The brains were cut sagittally through the midline, and one hemibrain was drop-fixed in $4 \%$ paraformaldehyde in PBS (pH 7.5) for $\sim 48 \mathrm{hrs}$ at $4^{\circ} \mathrm{C}$ followed by processing and paraffin embedding. The other hemibrain was snap frozen with isopentane on dry-ice and then stored at $-80 \otimes \mathrm{C}$ until it was thawed and homogenized in preparation for ELISA measurements of $A \beta$ peptide levels.

\section{Histology and immunochemistry}

Paraffin sections ( $5 \mu \mathrm{m})$ were used for all the histology and immunochemistry studies. Campbell-Switzer silver (34) and Thio-S (27) tissue staining methods were performed as previously described. Immunochemistry followed standard protocols described previously (27). To assess amyloid pathology, embedded sections were immunohistochemically stained with a biotinylated pan-A $\beta$ antibody Ab5 (1:500; T.E.G.) and developed using Vectastain Elite ABC Kit (Vector Laboratories, Burlingame, $C A$ ) followed with 3,3'-diaminobenzidine (DAB) substrate (Vector Laboratories, Burlingame, CA). To evaluate tau pathology, embedded sections were immunohistochemically stained with 7F2 (1:10,000; Ben Giasson (29)), CP27 (1:1000; Peter Davies), AT8 (1:1000; ThermoFisher Scientific), and MC1 (1:1000; Peter Davies). To assess aSyn pathology, sections were stained with 9C10 (1:1000; Ben Giasson) (20). After development by 3,3'diaminobenzidine (DAB) (Vector Laboratories, Burlingame, $C A$ ) substrate and counterstaining with hematoxylin, the slides were coverslipped and images were taken using an Olympus BX60 microscope or scanned by Aperio ${ }^{\circledR}$ XT System (Leica Biosystems, Buffalo Gove, IL, USA) or Zeiss microscope (Carl Zeiss, Oberkochen, Germany) and analyzed using the Zen 2.6 program (Carl Zeiss, Oberkochen, Germany). Blinded observers then reviewed the images and scored the burden of $A \beta$ pathology based on the following criteria; "+++" = heavy A $\beta$ burden with too many deposits to count. " ++ " = abundant pathology with $>30$ deposits per section. " + " = consistent pathology with $>5$ deposits per section. " $+/=$ inconsistent pathology with $<3$ deposits per section. 


\section{Brain amyloid extraction}

Human brain samples were originally analyzed by Moore (13). Briefly, frozen pre-frontal cortex tissue was cryo-pulverized in liquid nitrogen and then sequentially extracted with Tris-buffered saline (TBS), radioimmunoprecipitation buffer (RIPA), $2 \%$ sodium dodecyl sulfate (SDS), and $70 \%$ formic acid (FA) containing protease inhibitor cocktail (Roche, Indianapolis, IN, USA). After harvesting the mouse brain, the left hemisphere was flash-frozen in isopentane. The frozen cortex was sequentially extracted with protease inhibitor cocktail (Roche) containing Tris-buffered saline, RIPA buffer, $2 \%$ SDS, and $70 \%$ formic acid (FA) as described previously at a concentration of $150 \mathrm{mg} / \mathrm{ml}(13)$.

\section{ELISA}

A $\beta$ levels from the $2 \%$ SDS and 70\% FA extracted samples were quantified by sandwich ELISA as described previously (13). Total $A \beta$ was captured with mAb Ab9 and detected by HRP-conjugated mAb 4G8 (human AB17-24; Covance, Princeton, NJ, USA); A 1-40 was captured with monoclonal antibody (mAb) Ab9 (human AB1-16; T.E. Golde) and detected by horseradish peroxidase (HRP)-conjugated mAb

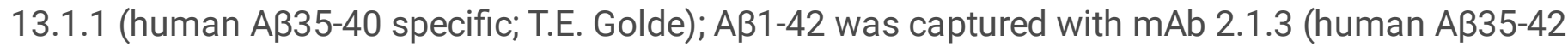
specific; T.E. Golde) and detected by HRP-conjugated mAb Ab9. ELISA results were analyzed using SoftMax Pro software (Molecular Devices).

\section{Contextual fear conditioning}

Transgenic PrP.APPsi and nontransgenic littermates, seeded with AD cases 1 and 2, and NDC case 7, were aged to 12 months and subjected to contextual fear conditioning as previously described $(35,36)$. Briefly, in the contextual fear conditioning test, a mouse learns the association between the training chamber, which represents an initially neutral conditional stimulus (CS) and an aversive, brief electric foot-shock, unconditional stimulus (US), which takes place in the training context. We have previously established that $0.45 \mathrm{~mA}$ electric current elicits robust avoidance response and results in strong freezing response after 2 CS-US pairings (35). During the training session, a mouse was allowed to explore the training chamber for $120 \mathrm{sec}$, with a $2 \mathrm{sec}$ foot shock immediately following a $30 \mathrm{sec}$ tone (80 dB) (day 1; see Fig. 4A). The mouse recovers for $60 \mathrm{sec}$ and then receives another $2 \mathrm{sec}$ foot shock following a 30 sec tone. The final post-US period is $60 \mathrm{sec}$. For the context test session, on day 3 , the mice were exposed to the same training context and the freezing presentation during the $300 \mathrm{sec}$ was recorded by an image analysis system (FreezeFrame, Actimetrics). On day 4 the mice experienced the tone session, they were placed in a modified chamber (altered inserts and smell), during the first $180 \mathrm{sec}$ the mouse was allowed to explore the new environment. During the second $180 \mathrm{sec}$ period the tone was delivered and the percent freezing was recorded. No shock was applied during both the test days.

\section{Results}

\section{Characterization of AD/LBD, AD, PA, and NDC cases}


In this study, we selected a subset of AD, PA, and NDC donors that were previously analyzed (13) for examination of seeding activity in APP, APP/tau, and tau transgenic mice (Table 1, AD 1 and 2, PA 1,2,3,4, and NDC 1 and 2). All 8 of these donors exhibited some degree of Lewy body pathology (LBD). Two of the donors selected for the study were sub-categorized with cerebral amyloid angiopathy (CAA); PA 3 had a high abundance of vascular amyloid with a CAA score of 2+-3+ while AD 1 had moderate levels of vascular $A \beta$ deposition with a score of $1+($ Table 1). As expected, the two $A D$ brains had significant accumulation of tau, indicated by Braak staging (5.5 and 6) (Table 1). The PA and NDC brains had lower abundance of tau than the $A D$ brains, but were similar to each other $(2,2.5,3,2$, and 2,3 , respectively) (Table 1). In this set of cases, all also exhibited incidental, diffuse, aSyn pathology. To these 8 , we added 6 additional donors that included two AD (no LBD), two with Lewy-body variant AD (AD/LBD), and 2 additional controls that were free of all types of pathology (Table 1, AD 3 and 4, AD/LBD 1 and 2, and NDC 3 and 4). These brains were used in seeding APP, APP/a-synuclein (aSyn), and aSyn mice so that we would be able to compare AD and NDC that lacked aSyn pathology to AD/LBD brains with high levels of aSyn pathology.

To characterize the pathology in the AD and PA brains used to seed the APP/tau, APP, and tau mice, sections were stained with a pan-A $\beta$ antibody (Fig. S1a), Thioflavin S (Thio-S) (Fig. S1b), and with a phospho-specific tau antibody (Fig. S1c). We observed widespread A $\beta$ deposition of both diffuse and compact amyloid in both AD and PA (Fig. S1a, b), with a subset showing striking CAA. PA 3 had numerous, Thio-S positive, vascular amyloid deposits, consistent with the assessment of CAA as 2+-3+ (Fig. S1a, b). Similarly, we observed Thio-S positive amyloid staining surrounding several vessels within AD 1, consistent with a CAA score of $1+$ (Fig. S1a, b). AD 2 contained several cored Thio-S positive deposits while the compact deposits in PA 1,2, and 4 had little to no Thio-S staining. As expected, AD 1 and 2 contained both substantial phosphorylated tau in form of dystrophic neurites, neuropil threads and neuronal inclusions while all four of the PA were negative for tau deposits (Fig. S1c). NDC 1 and 2 were negative for $A \beta$ and tau deposits by both immunostaining and Thio-S staining.

\section{Cerebral injection of brain lysates into newborn APP/tau and APP mice results in widespread, robust amyloid deposition}

To gain a better understanding of the type of $A \beta$ pathology that unseeded PrP.APPsi mice produce, we harvested breeder mice at various ages to assess phenotypic variation (Online Resource, Fig. S2a and b) (27). At 12 months of age, when deposition begins to occur in the brains of PrP.APPsi mice, any given section through the cortex and hippocampus may exhibit 1 or 2 diffuse tufted deposits and/or cored deposits (score +/-). At this age, $A \beta$ deposition also begins to appear in the meninges surrounding the cerebellum (Online Resource 2, Fig. S2a). Approximately 50\% of PrP.APPsi mice at 11-13 months of age exhibit no $A \beta$ pathology or show only meningeal deposition in the cerebellum (Online Resource 2, Fig. S2a). To concurrently examine whether tau pathology could be augmented by seeding from these lysates, 
the PrP.APPsi mice were crossed to a line of mice that express human tau P301L (iTau-P301L) (Table 2) $(28,37)$. The iTau-P301 L mice express mutant human tau at levels 2-3 fold higher than nontransgenic mice (Fig. S3).

To compare the relative ability of homogenates prepared from $A D$ and $P A$ cases to seed $A \beta$ deposition in these mice, lysates from the pre-frontal cortex of the $A D, P A$, and NDC were injected into the cerebral ventricles of newborn mice at PO $(20,32,33)$. We confirmed that the lysates contained $A \beta$ by immunoblotting and ELISA (Fig. S1d and Table 1). A $\beta$ was detectable by immunoblot in each of the lysates used for injection (Fig. S1d), and enzyme-linked immunosorbent assay (ELISA) measurements confirmed high levels of SDS-soluble and formic acid (FA) soluble A $\beta 42$ in each brain lysate (Table 1). We hypothesized that injection of these lysates in P0 mice would result in widespread dispersion of the A $\beta$ or tau seeds, and potentially offer the best chance to extensively alter the type of pathology that would be induced by seeding.

To assess amyloid pathology, hemibrains of seeded mice were stained with a pan-A $\beta$ antibody and Thio$S$. In mice injected with either $A D$ or PA lysates, we observed significant induction of $A \beta$ deposition, comprised primarily of diffuse deposits throughout the cortex and hippocampus, with some vascular deposition in the pia surrounding the cortex and within the hippocampal fissure (Fig. 1b, c). Mice injected with homogenates from NDC 1 and 2, and mice that were not injected, had little A $\beta$ pathology by 12 months of age (Fig. 1b, c). Examples of cases with the most severe pathology are shown in Figure 1, while images of animals with the least pathology are shown in Figure S4. In all cases, however, the induced $A \beta$ pathology exhibited a diffuse, Thio-S negative morphology (Fig. S5). The levels of induced $A \beta$ pathology in mice that co-expressed APPsi and Tau-P301L were similar to that of mice that expressed only APPsi (Fig. 1b versus c, S4a versus b). Mice that were transgenic for only Tau-P301L, or were nontransgenic, showed no evidence of $A \beta$ deposition (data not shown). To compare the data across all of the mice examined, $A \beta$ pathology was qualitatively scored by three independent observers (Fig. 1d, e). At 12 months, only PA 4, was scored as having a level of $A \beta$ pathology that approached mice injected with the $A D$ cases; both of which exhibited widespread, diffuse pathology (Fig. 1b, c). A $\beta$ pathology in mice seeded by PA 1, 2, and 3 was scored as less abundant than in mice seeded by the AD cases (Fig. 1d, e). We confirmed the high seeding activity of AD 1 and 2, and PA 4 by assessing A $\beta$ pathology at 9 months post-injection. Although the severity of pathology varied, multiple animals injected with each of these lysates exhibited $A \beta$ deposition (Fig. S6a). Notably, dilution of the brain lysates by 10 -fold prior to injection greatly diminished seeding activity (Fig. S6b). To further examine the relative seeding activity of PA lysates, we injected newborn PrP.APPsi mice with each of the 4 PA lysates and NDC 1, and analyzed pathology at 18 months post-injection. Although the mice injected with the PA lysates appeared to have more severe $A \beta$ deposition, we also observed some significant $A \beta$ pathology in animals injected with the NDC lysate (Fig S7). Whether this outcome was due to some small amount of A $\beta$ seed in the NDC 1 will require further study. Collectively, these studies demonstrated that $A D$ and PA brains contain misfolded forms of $A \beta$ that preferentially seed diffuse $A \beta$ pathology. 
Two of the donor cases were identified as having CAA pathology, AD 1 and PA 3 , and were scored $1+$ and $2+-3+$, respectively (Table 1 ). To determine if CAA pathology was seeded in the recipient mice, we stained sections with Thio-S and searched for evidence of vascular amyloid. In mice seeded with PA 3, we observed Thio-S positive, vascular pathology in 6 of the 7 recipient mice however, the incidence of this pathology was limited (Fig. S8). Although AD 1 also contained CAA pathology, we did not observe Thio-S positive, vascular pathology in mice seeded by this homogenate. These findings suggest that it may be possible to selectively increase CAA using seeding, but enhancing such pathology may require purification of cerebral vessels before preparation of the seeds.

We biochemically confirmed our histological data by analyzing $A \beta$ levels from sequentially extracted brain lysates using $C$-terminal specific antibodies in sandwich ELISAs to measure $A \beta 40$ and $A \beta 42$ specifically. In our $A \beta$ ELISAs the detection limit was approximately $0.04 \mathrm{pmol} / \mathrm{g}$. In the brains of all seeded mice, the levels of $A \beta 40$, both SDS-soluble and FA were 10-100 fold lower than that of $A \beta 42$ (Fig. 2a, b). Consistent with the presence of abundant diffuse $A \beta$ pathology, we detected elevated levels of $A \beta 42$ in SDS-soluble fractions from mice seeded with AD 1 and 2, and PA 4 (Fig. 2c). Somewhat surprisingly, the levels of $A \beta 42$ in the FA-soluble fractions were similar to that of the SDS fraction despite the absence of cored $A \beta$ deposits (Fig. $2 c$ and d). Notably, there was considerable variation in the levels of $A \beta$ in these seeded animals, which rendered relatively few indications of statistical differences (Fig. 2eh). For $A \beta 40$ measures, the only instance in which the seeded mice had levels that were higher than uninjected controls, or mice injected with NDC lysate, were mice seeded by AD 1 (Fig. 2e, f). For measurements of SDS-soluble A 42 , the brains of mice seeded with AD 1,2, and PA 4 were the only examples in which the levels were statistically higher than the levels in mice seeded with NDC 1 and 2, or uninjected mice (Fig. 2g). In the FA-soluble fractions, only the AD lysates possessed higher levels of Aß42 than the brains of mice seeded with the two NDC lysates or uninjected mice (Fig. 2h). Collectively, these findings demonstrated that both $A D$ and $P A$ brains have the potential to seed diffuse $A \beta$ pathology, with the AD brains appearing to be slightly more potent.

To determine whether the injection of these lysates into the brains of newborn PrP.APPsi/iTau-P301L mice also induced tau pathology, we stained the brains of the seeded animals with antibodies specific for phosphorylated tau (AT8) and misfolded tau (MC1). Immunostaining with the antibody CP27, which is specific for human tau, confirmed the presence of human P301L tau in the bigenic mice, but we observed no obvious reactivity with AT8 or MC1 antibodies (Fig. S9).

To determine if the induction of amyloid would secondarily induce aSyn pathology, we examined the seeding activity of individuals with $A D$ and LBD pathology. To characterize the pathology of these $A D$, $A D / L B D$ and NDC brains, we stained sections with a pan-A $\beta$ antibody (Fig. 3a), Thio-S (Fig. 3b), aSyn antibody (Fig. 3c), and a phospho-specific tau antibody (Fig. 3d). Both AD 3 and 4 contained numerous amyloid deposits that were compact and Thio-S positive (Fig. 3a, b), had widespread tau positive inclusions (Fig. 3d), and lacked aSyn pathology (Fig. 3c). AD 3 showed striking Thio-S positive amyloid staining surrounding vessels, consistent with a CAA score of $3+$ (Fig. 3b, Table 1). We also observed CAA staining with AD/LBD 1 (Fig. 3b). The AD/LBD cases showed modest A $\beta$ pathology with some aSyn 
pathology and sparse tau deposition (Fig. 3a, c, d). NDC 3 contained some amyloid pathology, but both lacked aSyn and tau pathology (Fig. 3).

PrP.APPsi/M20 and PrP.APPsi mice injected with AD brains developed widespread, robust A $\beta$ pathology by 12 months post-injection (Fig. $4 a$ and $b$ ). A $\beta$ deposition was primarily diffuse and Thio-S negative (Fig S10). There was no difference in A $\beta$ seeding capacity between PrP.APPsi/M20 and PrP.APPsi mice. Interestingly, $A D / L B D 1$ and 2 also promoted deposition of $A \beta$ in mice expressing APPsi with and without aSyn (Fig. 4), indicating that the AD/LBD brains contained considerable levels of $A \beta$ seeds despite a much lower burden of $A \beta$ pathology (Fig. 3). None of the injected animals developed appreciable aSyn pathology (data not shown). Although two of the donor cases contained significant CAA pathology, CAA was not seeded in the recipient mice (Fig S10).

\section{Seeding diffuse $A \beta$ deposition does not impair performance in a fear-conditioning cognitive task}

To assess whether the diffuse $A \beta$ pathology seeded by $A D$ brain produces cognitive deficits, an additional cohort of PrP.APPsi mice were seeded with AD 1 and 2, or NDC 1, and then were aged 12 months before behavioral testing in a fear-conditioning paradigm. Control groups were mice that were not injected with brain lysate and nontransgenic littermate mice that were either injected with the same lysates or uninjected. The performance of the animals in the contextual fear memory test was conducted twice. In the first round of testing, the performance of the seeded mice was not statistically different from that of the control uninjected mice (Fig. 5a). Overall, in this first round of testing, the percentage of time animals that exhibited freezing behavior was relatively low. In the second round of testing, we expected to reinforce memory of the adverse cue (context or tone) and we observed that re-tested mice showed increased levels of freezing behavior (Fig. 5b); however, there was still no statistically significant difference in the performance of the seeded mice relative to any control. These findings indicate that the diffuse $A \beta$ pathology seeded by these homogenates of human $A D$ brain does not produce a meaningful impairment in memory systems involved fear-conditioning memory tasks.

\section{Assessment of $A \beta$ seeding efficiency in APPsi mice.}

To examine the relative seeding efficiency of different types of A $\beta$ pathology in PrP.APPsi mice, we used an approach in which homogenates from the brains of 4 different lines of aged APP and APP/PS1 mice were used to seed accelerated $A \beta$ deposition (described in Table 2). At these advanced ages, each of the mice used to produce inoculum had substantial $A \beta$ pathology (Online Resource 2, Fig. S11). Following the strategy used above, we injected the homogenates containing $A \beta$ seeds into newborn mice on neonatal day 0 , which were then aged to 9,12 , or 15 months before euthanasia and neuropathological analysis by Campbell Switzer silver stain and Thio-S staining. The type of pathology noted and the relative abundance score are noted at the bottom of each column of images in Figure 6 . In all of the PrP.APPsi mice injected with brain homogenate seeds from mice that primarily exhibited cored plaques, we observed an obvious shift in the type of $A \beta$ deposits in recipient mice to match the pathology found in 
the donor mice used for seeding (Fig. 6). Self-seeding of newborn PrP.APPsi mice with brain homogenates from aged PrP.APPsi mice produced diffuse deposits (Fig. 6), which was the predominant form of $A \beta$ pathology in the PrP.APPsi animal used to generate the seeding homogenate (Online Resource 2 , Fig. S11). Similarly, the predominant pathology in the $\sim 25$ month old Tet.MoA $\beta$ animal used to prepare seeding homogenate was diffuse $A \beta$ deposits (Online Resource 2, Fig. S11)(38), and brain homogenates from this animal efficiently seeded the deposition of human $A \beta$ in the PrP.APPsi mice to produce diffuse deposits (Fig. 6). PrP.APPsi mice seeded with homogenates from donors that had primarily cored deposits had lower $A \beta$ burden scores, but importantly we observed a shift in the neuropathologic features of the recipient mice to a much higher incidence of cored deposits (Fig. 6). Interestingly, the morphology of deposits in the seeded PrP.APPsi mice was not quite identical to the source PrP.HuAß/PS1mice in that the seeded deposits were smaller and appeared to be more compact than the donor line. These small dense deposits were Thio-S positive (Fig. 6). Similar small dense core deposits that were Thio-S positive were observed in the cortex of PrP.APPsi mice injected with brain homogenates from the PrP.MoAB/PS1 mice (Fig. 6). Collectively, these findings demonstrate the relative ease with which diffuse $A \beta$ pathology can be induced in PrP.APPsi mice.

\section{Discussion}

We investigated whether injection of neonatal APP, APP/tau, or APP/aSyn mice with AD or AD/LBD brain homogenates could be used as a paradigm to generate mice that model the mixed pathology associated with each disease. Despite the widespread induction of diffuse $A \beta$ deposition by injection of four different AD brain lysates, and the presence of tau seeds in these lysates, tau pathology was not induced. For comparison, we also injected PA brain lysates, finding a similar induction of diffuse $A \beta$ deposition without induction of tau pathology. Similarly, injection of $A D / L B D$ brain lysates induced diffuse $A \beta$ deposition, but aSyn pathology was not induced. In all cases, the induced $A \beta$ pathology exhibited a diffuse, Thio-S negative, morphology. PrP.APPsi mice injected with AD brain lysates that develop diffuse $A \beta$ pathology showed no significant impairment in a fear-conditioning cognitive task. Our findings suggest that seeding the brains of neonatal transgenic mice with $A D, A D / L B D$, and PA brain homogenates can efficiently induce diffuse $A \beta$ deposition but neither $A D$ nor $A D / L B D$ brains seeded concurrent tau or aSyn pathology.

The most common route of administering $A \beta$ seeding preparations is by stereotaxic injection into the hippocampus of adult APP transgenic mice [reviewed in (3)]. In host mice that develop amyloid pathology at relatively young ages, the induced pathology created by seeding may be localized to the site of injection and overlying cortex (3); whereas, in models that do not develop deposits until late in life, the induced pathology may be more wide-spread [reviewed in (3)]. The PrP.APPsi host we used in the present study develops pathology on its own between 12-14 months of age. In testing the approach of injecting $A \beta$ seeds into newborn mice from this model, we aspired to attain a widespread distribution of seeding material and induce $A \beta$, tau, or aSyn pathology throughout the brain. This approach replicates previous studies where PO injection of adeno-associated virus resulted in widespread distribution in the brain (39). Injection of newborn hamsters with scrapie prions has been shown to accelerate onset of prion disease 
(40). Additionally, we have observed that intracerebral injection of newborn A53T aSyn mice with brain lysates from multiple system atrophy donors induces aSyn pathology and motor impairment (20), and that intraspinal injection of newborn mice expressing mutant superoxide dismutase (SOD1) can accelerate the onset of paralysis and pathology $(41,42)$. Thus, in performing newborn injections our goal was to initiate pathological cascades as early as possible in order to determine whether there may be distinct synergies between $A \beta$ and tau, or $A \beta$ and aSyn, which can be elaborated by seeding.

We recognize that there are a large number of potential mouse models that could have been used for these studies. The APP mouse model we chose to us for these studies was selected because PrP.APPsi mice do not inherently develop $A \beta$ pathology until 12-14 months of age and because this model can exhibit a full spectrum of $A \beta$ pathology including cored-neuritic, diffuse, and vascular deposition (see Supplemental Fig. S2). The tau model we chose to use expresses human P301L tau at relatively low levels and does not develop tau pathology on its own. When paired with mice that express the tetracycline transactivator in the $\mathrm{rTg} 4510$ model these develop a robust tau pathology (28). In paradigms in which tau expression in rTg4510 mice is induced early and then suppressed by doxycycline, the low level of "leaky" tau expression in this model is sufficient to sustain neurofibrillary tangle pathology (28). In our paradigm, we asked whether neonatal seeding of this tau model could induce a sustained pathology in the same way that early expression of the transgene at high levels produced sustained pathology. Alternatively, we were interested to determine whether we could detect any synergy between concurrent $A \beta$ pathology and tau seeding. The aSyn model we chose to use, expresses human WT aSyn at levels that do not cause pathology (31). WT aSyn (M20) mice can be seeded when injected with high levels of purified aSyn fibrils, but are not easily seeded by human brain homogenates (20). Our goal in choosing the M20 WT aSyn mice was to develop a model of human aSyn pathology and assess whether concurrent $A \beta$ pathology could synergize aSyn seeding.

Our method of seed preparation followed commonly used protocols where we injected a soluble fraction; frontal cortex homogenized in PBS $(10 \% \mathrm{w} / \mathrm{v})$ followed by sonication and low speed centrifugation, as previously described $(32,43,44)$. By this method, we expected sonication to fragment all types of seeds with a mixture of seeds remaining in suspension after low-speed centrifugation. As we have observed here, the most common outcome of $A \beta$ seeding with similar preparations is induction of diffuse $A \beta$ pathology [reviewed in (3)].

In order to efficiently seed mixed pathology, it may be necessary to optimize preparations of each type of seed independently. Interestingly, injection of the supernatant and pellet of fractionated APP23 brain homogenate resulted in morphologically different $A \beta$ deposits, with the supernatant fraction seeding diffuse, Congo Red negative $A \beta$ and the pellet seeding deposits similar to the total lysate, a mixture of diffuse and punctate, Congo Red positive deposits (45). Several studies have shown induction of tau pathology after injection of synthetic tau fibrils, brain extract from mutant P301S tau mouse, or human AD, corticobasal degeneration, and progressive supranuclear palsy brain lysates (46-52). In these studies, the human brain lysates were enriched for tau seeds by sequential fractionation with the addition of sucrose and/or sarkosyl, creating fractions that contain potent tau seeds. Similarly, injection of the 
fractionated homogenate from brain lysates of individuals with LBD resulted in the induction of aSyn, indicating that with enrichment it is possible to seed aSyn pathology directly from human brain $(8,53$, 54). Because one of our goals was to determine whether we could detect synergy between $A \beta$ and tau or aSyn pathology, we chose to avoid enriching for any particular type of seeding activity. The absence of tau or aSyn pathology in our seeded models indicates that the type of $A \beta$ deposition we generated did not synergize to induce, or exacerbate, tau or aSyn pathology.

We demonstrated that the $A \beta$ seeding activity of brain lysates from PA cases was similar to $A D$ and $A D / L B D$ cases. Although mice seeded by PA brains scored as having less amyloid positivity at 12 months post-injection than mice injected with AD brain lysates, the difference between PA and AD brain seeding activity was less evident by 18 months post-injection. Our study was not powered or designed to determine whether homogenates from $A D$ and PA brains have quantitative differences in seeding activity. All three of the PA cases tested were able to seed amyloid pathology, supporting the hypothesis that the $A \beta$ pathology in PA is similar to $A D(13)$.

Interestingly, several of the brain lysates we used were from tissues that exhibited relatively high levels of CAA pathology (see Table 1). PA 3 and AD 3 were both scored as having the highest levels of CAA, with significant CAA in AD/LBD 1 and 2. In mice injected with PA 3, we observed modest seeding of parenchymal CAA pathology in the host mice but in all other mice the only obvious vascular deposition was meningeal. Augmented CAA pathology has been observed in APP23 mice, which develop CAA, by intraperitoneal injection of APP23 lysates (55). CAA pathology was also induced in CRND8 mice by injection of synthetic $A \beta 42$ oligomers, generated in the presence of anionic micelles composed of fatty acids (56). Recent studies have documented early onset cerebrovascular $A \beta$ pathology in individuals receiving dura mater transplants, suggesting that CAA may be transmitted iatrogenically (57). Collectively, these results suggest that CAA could be a distinct conformer of $A \beta$ that may be independently propagated.

Not all $A \beta$ conformers seeded as proficiently as vascular $A \beta$. Tissues from $A D$ and $A D / L B D$ brains that were used to prepare these lysates contained both diffuse and compact $A \beta$ deposits, with significant levels of formic acid extractable $A \beta$. Yet, even in mice aged to 18 months of age, diffuse $A \beta$ deposits were the dominant form of pathology. These results mirror similar studies in which injection of human brain homogenates resulted in induction of diffuse $A \beta$ pathology, with relatively few ThioS or congophilic compact deposits $(17,39,58,59)$. The lack of conversion of the diffuse deposits to cored plaques indicates different types of amyloid deposition are not freely interchangeable, supporting the idea that different types of $A \beta$ pathology arise from different amyloid strains with distinct seeding capabilities. This conclusion is also supported by our studies of $A \beta$ seeding in which transgenic mice were used as the seed source. Newborn PrP.APPsi mice injected with transgenic mouse brain homogenates prepared from mice that exhibit high levels of diffuse $A \beta$ pathology produced a robust induction of diffuse $A \beta$ deposition by 12 months post-injection. By contrast, brain homogenates prepared from mice that predominantly produce cored, Thio-S positive, $A \beta$ deposits induced minimal $A \beta$ deposition by 12 months. These results are consistent with previous studies with APP/PS1 and APP23 mice. Injection of APPPS1 
mice that typically develop compact, punctate plaques, with APP23 seeds results in a mixture of diffuse, filamentous $A \beta$ as well as compact plaques $(32,60)$. Injection of APP23 mice that typically develop mixed pathology, both core and diffuse plaques, with APPPS1 homogenate results in plaques that are more diffuse than the punctate deposits seen in APPPS1 mice injected with APPPS1 homogenate (32, $60)$. Thus, although it is possible to seed cored deposits in APP mice $(32,45,61-63)$, it appears that diffuse $A \beta$ pathology is more easily seeded. Together these results suggest that $A \beta$ plaques differ in their morphology, seeding ability, and impact on cognitive function. Current amyloid therapies aim to reduce amyloid deposition without much consideration of the type of plaque that is being targeted. Reduction of diffuse amyloid may at first appear to be beneficial, since it appears to be the dominate seeding strain, however, it may not be the most pathological strain. Cognitive behavior studies of the seeded APPsi mice reported here, and of a non-seeded Bri-A $\beta 42$ model that also shows primarily diffuse A $\beta$ pathology (64), failed to associate diffuse amyloid with reduced performance in a fear-conditioning memory task. If diffuse $A \beta$ pathology is less damaging, then therapies that only reduce diffuse amyloid would not yield much therapeutic benefit.

\section{Limitations}

There are many potential transgenic mouse models available that could be used for the type of seeding studies we conducted. The APP model we used appears to have performed as expected, but the lack of tau or aSyn seeding may be a consequence of the host model chosen to express human tau and aSyn or the dose of the tau and aSyn seeds in the seeding homogenate. The expression of human P301L tau in the tet-tau model used here may have been too low to sustain propagation of tau seeds. In the time between when these studies were initiated and publication, a study of seeding in the JNPL3 model of human P301L tau, which expresses tau at levels similar to the tet-tau model used here, reported poor induction of tau pathology after seeding (36). A higher dose of enriched tau seeds may have been more effective. Likewise, a higher dose of aSyn seeds may be required to induce human WT aSyn to produce pathology. Our study demonstrates that using seeding to produce novel models of mixed pathology will require further refinement in methodology to achieve the desired outcome.

\section{Conclusions}

In conclusion, we assessed the relative seeding activity of brain lysates prepared from $A D, A D / L B D$, and PA brain by injecting neonatal transgenic hosts. We found that PO injection of transgenic mice expressing humanized APP with $A \beta$-containing lysates, resulted in robust and widespread induction of $A \beta$ pathology. The induced $A \beta$ pathology was diffuse, which has been reported in other seeding studies where adult animals were the recipients of injections (3). Our results are consistent with earlier reports in finding that diffuse $A \beta$ is easily seeded by crude homogenates of human brain. Seeds for diffuse $A \beta$ were prevalent in human $A D, A D / L B D$, and PA brain. The diffuse $A \beta$ pathology induced in these models was not accompanied by secondary tau or aSyn pathology in bigenic mice co-expressing APP/tau or APP/aSyn. Mice with seeded diffuse $A \beta$ pathology were not impaired in a fear-conditioning memory task. Further 
refinements in methodology will be required to efficiently seed models of mixed $A D$ and $A D / L B D$ pathology.

\section{Abbreviations}

AD: Alzheimer's disease

LBD: Lewy body disease

AD/LBD: Alzheimer's disease/Lewy body disease

$A \beta$ : amyloid- $\beta$

aSyn: a-synuclein

APP: Amyloid precursor protein

PA: Pathological Aging

GFAP: Glial fibrillary acidic protein

MAPT: Microtubule-associated protein tau

NDC: Nondemented Controls

WT: Wild type

CAA: cerebral amyloid angiopathy

ELISA: Enzyme linked immunosorbent assay

SDS: Sodium dodecyl sulfate

FA: Formic acid

Thio-S: Thioflavin S

\section{Declarations}

\section{Ethics approval}

Human brain tissue was obtained from the Mayo Clinic Brain Bank and University of Florida Neuromedicine Human Brain Tissue Bank with informed consent following institutional regulations. All animal procedures were approved by the University of Florida Institutional Animal Care and Use Committee. 


\section{Consent for publication}

Not Applicable

\section{Availability of data and materials}

All data generated and analyzed during this study are included in this manuscript.

\section{Competing interests}

The authors declare that they have no competing interests.

\section{Funding}

This work was supported by funding from the National Institute of Health; grant numbers R21AG046125, R21AG055113, U54NS110435, P50AG047266, P30AG066506.

\section{Authors' contributions}

Study concept and design: BDM, YL, JL, BIG, DRB. Pathological analysis: SP and DD. Acquisition of data: BDM, YL, GX, HH, MFA, CLC, HSF, CM, SF, SP, DD. Statistical analysis: BDM and DRB. Analysis and interpretation of the data: BDM, YL, GX, CJ, JL, BIG, TEG, DRB. Drafting of the manuscript: BDM and DRB. Acquisition of funding: JL, BIG, TEG, DRB. All authors read and approved the final manuscript.

\section{Acknowledgements}

The authors would like to thank the patients and their families that donated tissue. We thank Dr. Peter Davies (Feinstein Institute for Medical Research, Manhasset, NY, USA) posthumously for antibodies to tau.

\section{Conflict of interest}

TEG is a co-founder of Lacerta Therapeutics

\section{References}

1. Hardy JA, Higgins GA. Alzheimer's disease: the amyloid cascade hypothesis. Science. 1992;256(5054):184-5.

2. Selkoe DJ, Hardy J. The amyloid hypothesis of Alzheimer's disease at 25 years. EMBO Mol Med. 2016;8(6):595-608.

3. Ulm BS, Borchelt DR, Moore BD. Remodeling Alzheimer-amyloidosis models by seeding. Mol Neurodegener. 2021;16(1):8.

4. Vergara C, Houben S, Suain V, Yilmaz Z, De Decker R, Vanden Dries V, et al. Amyloid-beta pathology enhances pathological fibrillary tau seeding induced by Alzheimer PHF in vivo. Acta Neuropathol. 
2019;137(3):397-412.

5. Pletnikova O, West N, Lee MK, Rudow GL, Skolasky RL, Dawson TM, et al. Abeta deposition is associated with enhanced cortical alpha-synuclein lesions in Lewy body diseases. Neurobiol Aging. 2005;26(8):1183-92.

6. Irwin DJ, Lee VM, Trojanowski JQ. Parkinson's disease dementia: convergence of alpha-synuclein, tau and amyloid-beta pathologies. Nat Rev Neurosci. 2013;14(9):626-36.

7. Lloyd GM, Dhillon JS, Gorion KM, Riffe C, Fromholt SE, Xia Y, et al. Collusion of alpha-Synuclein and Abeta aggravating co-morbidities in a novel prion-type mouse model. Mol Neurodegener. 2021;16(1):63.

8. Bassil F, Brown HJ, Pattabhiraman S, Iwasyk JE, Maghames CM, Meymand ES, et al. Amyloid-Beta (Abeta) Plaques Promote Seeding and Spreading of Alpha-Synuclein and Tau in a Mouse Model of Lewy Body Disorders with Abeta Pathology. Neuron. 2020;105(2):260-75 e6.

9. Spencer B, Desplats PA, Overk CR, Valera-Martin E, Rissman RA, Wu C, et al. Reducing Endogenous alpha-Synuclein Mitigates the Degeneration of Selective Neuronal Populations in an Alzheimer's Disease Transgenic Mouse Model. J Neurosci. 2016;36(30):7971-84.

10. Yamada M, Tsukagoshi H, Otomo E, Hayakawa M. Systemic amyloid deposition in old age and dementia of Alzheimer type: the relationship of brain amyloid to other amyloid. Acta Neuropathol. 1988;77(2):136-41.

11. Golde TE, Schneider LS, Koo EH. Anti-abeta therapeutics in Alzheimer's disease: the need for a paradigm shift. Neuron. 2011;69(2):203-13.

12. Jack CR, Jr., Lowe VJ, Weigand SD, Wiste HJ, Senjem ML, Knopman DS, et al. Serial PIB and MRI in normal, mild cognitive impairment and Alzheimer's disease: implications for sequence of pathological events in Alzheimer's disease. Brain. 2009;132(Pt 5):1355-65.

13. Moore BD, Chakrabarty P, Levites Y, Kukar TL, Baine AM, Moroni T, et al. Overlapping profiles of Abeta peptides in the Alzheimer's disease and pathological aging brains. Alzheimers Res Ther. 2012;4(3):18.

14. Dickson DW, Crystal HA, Mattiace LA, Masur DM, Blau AD, Davies P, et al. Identification of normal and pathological aging in prospectively studied nondemented elderly humans. Neurobiol Aging. 1992;13(1):179-89.

15. Dickson DW, Crystal HA, Bevona C, Honer W, Vincent I, Davies P. Correlations of synaptic and pathological markers with cognition of the elderly. Neurobiol Aging. 1995;16(3):285-98; discussion 98-304.

16. Golde TE, Dickson D, Hutton M. Filling the gaps in the abeta cascade hypothesis of Alzheimer's disease. Curr Alzheimer Res. 2006;3(5):421-30.

17. Kane MD, Lipinski WJ, Callahan MJ, Bian F, Durham RA, Schwarz RD, et al. Evidence for seeding of beta -amyloid by intracerebral infusion of Alzheimer brain extracts in beta -amyloid precursor proteintransgenic mice. J Neurosci. 2000;20(10):3606-11. 
18. Ayers JI, Fromholt S, Sinyavskaya O, Siemienski Z, Rosario AM, Li A, et al. Widespread and efficient transduction of spinal cord and brain following neonatal AAV injection and potential disease modifying effect in ALS mice. Mol Ther. 2015;23(1):53-62.

19. Kim JY, Grunke SD, Levites Y, Golde TE, Jankowsky JL. Intracerebroventricular viral injection of the neonatal mouse brain for persistent and widespread neuronal transduction. $\mathrm{J}$ Vis Exp. 2014(91):51863.

20. Dhillon JS, Trejo-Lopez JA, Riffe C, Levites Y, Sacino AN, Borchelt DR, et al. Comparative analyses of the in vivo induction and transmission of alpha-synuclein pathology in transgenic mice by MSA brain lysate and recombinant alpha-synuclein fibrils. Acta Neuropathol Commun. 2019;7(1):80.

21. Hyman BT, Phelps CH, Beach TG, Bigio EH, Cairns NJ, Carrillo MC, et al. National Institute on AgingAlzheimer's Association guidelines for the neuropathologic assessment of Alzheimer's disease. Alzheimers Dement. 2012;8(1):1-13.

22. Saido TC, Kawashima S, Tani E, Yokota M. Up- and down-regulation of calpain inhibitor polypeptide, calpastatin, in postischemic hippocampus. Neurosci Lett. 1997;227(2):75-8.

23. Schmidt ML, DiDario AG, Otvos L, Jr., Hoshi N, Kant JA, Lee VM, et al. Plaque-associated neuronal proteins: a recurrent motif in neuritic amyloid deposits throughout diverse cortical areas of the Alzheimer's disease brain. Exp Neurol. 1994;130(2):311-22.

24. McKeith IG, Boeve BF, Dickson DW, Halliday G, Taylor JP, Weintraub D, et al. Diagnosis and management of dementia with Lewy bodies: Fourth consensus report of the DLB Consortium. Neurology. 2017;89(1):88-100.

25. Montine TJ, Phelps CH, Beach TG, Bigio EH, Cairns NJ, Dickson DW, et al. National Institute on AgingAlzheimer's Association guidelines for the neuropathologic assessment of Alzheimer's disease: a practical approach. Acta Neuropathol. 2012;123(1):1-11.

26. Charidimou A, Martinez-Ramirez S, Shoamanesh A, Oliveira-Filho J, Frosch M, Vashkevich A, et al. Cerebral amyloid angiopathy with and without hemorrhage: evidence for different disease phenotypes. Neurology. 2015;84(12):1206-12.

27. Xu G, Ran Y, Fromholt SE, Fu C, Yachnis AT, Golde TE, et al. Murine Abeta over-production produces diffuse and compact Alzheimer-type amyloid deposits. Acta Neuropathol Commun. 2015;3:72.

28. Santacruz K, Lewis J, Spires T, Paulson J, Kotilinek L, Ingelsson M, et al. Tau suppression in a neurodegenerative mouse model improves memory function. Science. 2005;309(5733):476-81.

29. Strang KH, Goodwin MS, Riffe C, Moore BD, Chakrabarty P, Levites Y, et al. Generation and characterization of new monoclonal antibodies targeting the PHF1 and AT8 epitopes on human tau. Acta Neuropathol Commun. 2017;5(1):58.

30. Xia Y, Bell BM, Giasson BI. Tau K321/K353 pseudoacetylation within KXGS motifs regulates taumicrotubule interactions and inhibits aggregation. Sci Rep. 2021;11(1):17069.

31. Giasson BI, Duda JE, Quinn SM, Zhang B, Trojanowski JQ, Lee VM. Neuronal alpha-synucleinopathy with severe movement disorder in mice expressing A53T human alpha-synuclein. Neuron. 2002;34(4):521-33. 
32. Meyer-Luehmann M, Coomaraswamy J, Bolmont T, Kaeser S, Schaefer C, Kilger E, et al. Exogenous induction of cerebral beta-amyloidogenesis is governed by agent and host. Science. 2006;313(5794):1781-4.

33. Chakrabarty P, Rosario A, Cruz P, Siemienski Z, Ceballos-Diaz C, Crosby K, et al. Capsid serotype and timing of injection determines AAV transduction in the neonatal mice brain. PLoS One. 2013;8(6):e67680.

34. Vallet PG, Guntern R, Hof PR, Golaz J, Delacourte A, Robakis NK, et al. A comparative study of histological and immunohistochemical methods for neurofibrillary tangles and senile plaques in Alzheimer's disease. Acta Neuropathol. 1992;83(2):170-8.

35. Hanna A, Iremonger K, Das P, Dickson D, Golde T, Janus C. Age-related increase in amyloid plaque burden is associated with impairment in conditioned fear memory in CRND8 mouse model of amyloidosis. Alzheimers Res Ther. 2012;4(3):21.

36. Chakrabarty P, Hudson VJ, III, Sacino AN, Brooks MM, D'Alton S, Lewis J, et al. Inefficient induction and spread of seeded tau pathology in P301L mouse model of tauopathy suggests inherent physiological barriers to transmission. Acta Neuropathol. 2015;130(2):303-5.

37. Lewis J, McGowan E, Rockwood J, Melrose H, Nacharaju P, Van Slegtenhorst M, et al. Neurofibrillary tangles, amyotrophy and progressive motor disturbance in mice expressing mutant (P301L) tau protein. Nat Genet. 2000;25(4):402-5.

38. Xu G, Fromholt SE, Chakrabarty P, Zhu F, Liu X, Pace MC, et al. Diversity in Abeta deposit morphology and secondary proteome insolubility across models of Alzheimer-type amyloidosis. Acta Neuropathol Commun. 2020;8(1):43.

39. Walker LC, Callahan MJ, Bian F, Durham RA, Roher AE, Lipinski WJ. Exogenous induction of cerebral beta-amyloidosis in betaAPP-transgenic mice. Peptides. 2002;23(7):1241-7.

40. McKinley MP, DeArmond SJ, Torchia M, Mobley WC, Prusiner SB. Acceleration of scrapie in neonatal Syrian hamsters. Neurology. 1989;39(10):1319-24.

41. Ayers JI, Fromholt S, Koch M, DeBosier A, McMahon B, Xu G, et al. Experimental transmissibility of mutant SOD1 motor neuron disease. Acta Neuropathol. 2014;128(6):791-803.

42. Bidhendi EE, Bergh J, Zetterstrom P, Andersen PM, Marklund SL, Brannstrom T. Two superoxide dismutase prion strains transmit amyotrophic lateral sclerosis-like disease. J Clin Invest. 2016;126(6):2249-53.

43. Eisele YS, Bolmont T, Heikenwalder M, Langer F, Jacobson LH, Yan ZX, et al. Induction of cerebral beta-amyloidosis: intracerebral versus systemic Abeta inoculation. Proc Natl Acad Sci U S A. 2009;106(31):12926-31.

44. Ziegler-Waldkirch S, d'Errico P, Sauer JF, Erny D, Savanthrapadian S, Loreth D, et al. Seed-induced Abeta deposition is modulated by microglia under environmental enrichment in a mouse model of Alzheimer's disease. EMBO J. 2018;37(2):167-82.

45. Langer F, Eisele YS, Fritschi SK, Staufenbiel M, Walker LC, Jucker M. Soluble Abeta seeds are potent inducers of cerebral beta-amyloid deposition. J Neurosci. 2011;31(41):14488-95. 
46. Peeraer E, Bottelbergs A, Van Kolen K, Stancu IC, Vasconcelos B, Mahieu M, et al. Intracerebral injection of preformed synthetic tau fibrils initiates widespread tauopathy and neuronal loss in the brains of tau transgenic mice. Neurobiol Dis. 2015;73:83-95.

47. Clavaguera F, Bolmont T, Crowther RA, Abramowski D, Frank S, Probst A, et al. Transmission and spreading of tauopathy in transgenic mouse brain. Nat Cell Biol. 2009;11(7):909-13.

48. Guo JL, Narasimhan S, Changolkar L, He Z, Stieber A, Zhang B, et al. Unique pathological tau conformers from Alzheimer's brains transmit tau pathology in nontransgenic mice. J Exp Med. 2016;213(12):2635-54.

49. Guo JL, Lee VM. Neurofibrillary tangle-like tau pathology induced by synthetic tau fibrils in primary neurons over-expressing mutant tau. FEBS Lett. 2013;587(6):717-23.

50. Boluda S, Iba M, Zhang B, Raible KM, Lee VM, Trojanowski JQ. Differential induction and spread of tau pathology in young PS19 tau transgenic mice following intracerebral injections of pathological tau from Alzheimer's disease or corticobasal degeneration brains. Acta Neuropathol. 2015;129(2):221-37.

51. Narasimhan S, Guo JL, Changolkar L, Stieber A, McBride JD, Silva LV, et al. Pathological Tau Strains from Human Brains Recapitulate the Diversity of Tauopathies in Nontransgenic Mouse Brain. J Neurosci. 2017;37(47):11406-23.

52. Clavaguera F, Akatsu H, Fraser G, Crowther RA, Frank S, Hench J, et al. Brain homogenates from human tauopathies induce tau inclusions in mouse brain. Proc Natl Acad Sci U S A. 2013;110(23):9535-40.

53. Masuda-Suzukake M, Nonaka T, Hosokawa M, Oikawa T, Arai T, Akiyama H, et al. Prion-like spreading of pathological alpha-synuclein in brain. Brain. 2013;136(Pt 4):1128-38.

54. Recasens A, Dehay B, Bove J, Carballo-Carbajal I, Dovero S, Perez-Villalba A, et al. Lewy body extracts from Parkinson disease brains trigger alpha-synuclein pathology and neurodegeneration in mice and monkeys. Ann Neurol. 2014;75(3):351-62.

55. Eisele YS, Obermuller U, Heilbronner G, Baumann F, Kaeser SA, Wolburg H, et al. Peripherally applied Abeta-containing inoculates induce cerebral beta-amyloidosis. Science. 2010;330(6006):980-2.

56. Saha J, Dean DN, Dhakal S, Stockmal KA, Morgan SE, Dillon KD, et al. Biophysical characteristics of lipid-induced Abeta oligomers correlate to distinctive phenotypes in transgenic mice. FASEB J. 2021;35(2):e21318.

57. Preusser M, Strobel T, Gelpi E, Eiler M, Broessner G, Schmutzhard E, et al. Alzheimer-type neuropathology in a 28 year old patient with iatrogenic Creutzfeldt-Jakob disease after dural grafting. J Neurol Neurosurg Psychiatry. 2006;77(3):413-6.

58. Morales R, Duran-Aniotz C, Castilla J, Estrada LD, Soto C. De novo induction of amyloid-beta deposition in vivo. Mol Psychiatry. 2012;17(12):1347-53.

59. Fritschi SK, Langer F, Kaeser SA, Maia LF, Portelius E, Pinotsi D, et al. Highly potent soluble amyloidbeta seeds in human Alzheimer brain but not cerebrospinal fluid. Brain. 2014;137(Pt 11):2909-15. 
60. Heilbronner G, Eisele YS, Langer F, Kaeser SA, Novotny R, Nagarathinam A, et al. Seeded strain-like transmission of beta-amyloid morphotypes in APP transgenic mice. EMBO Rep. 2013;14(11):101722.

61. Eisele YS, Fritschi SK, Hamaguchi T, Obermuller U, Fuger P, Skodras A, et al. Multiple factors contribute to the peripheral induction of cerebral beta-amyloidosis. J Neurosci. 2014;34(31):1026473.

62. Fritschi SK, Cintron A, Ye L, Mahler J, Buhler A, Baumann F, et al. Abeta seeds resist inactivation by formaldehyde. Acta Neuropathol. 2014;128(4):477-84.

63. Ye L, Rasmussen J, Kaeser SA, Marzesco AM, Obermuller U, Mahler J, et al. Abeta seeding potency peaks in the early stages of cerebral beta-amyloidosis. EMBO Rep. 2017;18(9):1536-44.

64. Kim J, Chakrabarty P, Hanna A, March A, Dickson DW, Borchelt DR, et al. Normal cognition in transgenic BRI2-Abeta mice. Mol Neurodegener. 2013;8:15.

\section{Tables}

Table 1. List of human brain samples used in this study.

\begin{tabular}{|c|c|c|c|c|c|c|c|c|c|c|c|c|c|}
\hline Case ID & Path $\mathrm{Dx}$ & LBD & Thal phase & Braak stage & CERA D \& Ore & SDS $A B 40$ & SDS-A342 & $\mathrm{FA}-\mathrm{AB} 40$ & $F A \rightarrow A B 42$ & CAA & APOE & Gender & $A g 9$ \\
\hline $\mathrm{AD} 1$ & $\mathrm{AD}$ (imt)LBD & alluse & 5 & $\mathrm{~V}$ & sparse & 7.23 & 201.03 & 334.81 & 967.13 & $1+$ & $e 3 / 64$ & $m$ & 84 \\
\hline $\mathrm{AD} 2$ & $A D$ (int) $B D$ & omse & 5 & VNI & sparse & $x$ & 106.95 & 49407 & 882.87 & 0 & $2 / \mathrm{le} 3$ & $f$ & 84 \\
\hline PA 1 & PALBD & almuse & 3 & 1 & none & 4.48 & 48.84 & 35.22 & 892.84 & 0 & $\mathrm{e} 3 / \mathrm{e} 4$ & $m$ & 78 \\
\hline PA2 & PALBD & limbic & 3 & III & none & 7.48 & 188.15 & $x$ & 319.68 & 0 & $03 / 64$ & $f$ & 83 \\
\hline PA3 & PALBD & ormase & 3 & II & none & 9.60 & 50.99 & 3821.32 & 986.61 & $2+3 *$ & $04 / 64$ & $\mathrm{~m}$ & 80 \\
\hline PA4 & PALBDIOA/tr & aimuse & 3 & 1 & none & 6.61 & 167.48 & 69.15 & 1261.48 & 0 & $e 03 / 63$ & $\mathrm{~m}$ & 81 \\
\hline NDC 1 & LBDIGBA & Imbic & 0 & 1 & nane & $x$ & $x$ & 21.43 & 5.72 & 0 & $2 / \mathrm{le} 3$ & $f$ & 78 \\
\hline $\mathrm{NDC} 2$ & $\angle B D$ & omse & 1 & II & none & 1.32 & 1.86 & $x$ & $x$ & 0 & $20 / 03$ & $m$ & 75 \\
\hline & & & & & & & & & & & & & \\
\hline $\mathrm{AD} 3$ & $\mathrm{AD}$ (high) & & 5 & $\mathrm{~V}$ & frequert & ND & $\mathrm{ND}$ & ND & $\mathrm{ND}$ & $2+$ & $63 / 64$ & $f$ & 86 \\
\hline $\mathrm{AD} 4$ & $A D(\mathrm{mt})$ & & 3 & V & moderate & ND & ND & ND & $\mathrm{ND}$ & $1+2+$ & $e 3 / 63$ & $\mathrm{~m}$ & 82 \\
\hline$A D M B D 1$ & $A D$ irf $1 B D$ & dilluse & 3 & $\mathrm{~V}$ & frequert & ND & $\mathrm{ND}$ & ND & $\mathrm{ND}$ & $2+$ & $63 / 64$ & $\mathrm{~m}$ & 83 \\
\hline ADILBD 2 & AD (high)/LDD & dilluse & 5 & N & frequert: & ND & ND & ND & ND & $2+$ & $e 3 / 63$ & $f$ & 81 \\
\hline NDC 3 & $\mathrm{AD}$ (bw) & & 3 & 1 & none & ND & $\mathrm{ND}$ & ND & $\mathrm{ND}$ & 0 & $\mathrm{ND}$ & $m$ & 52 \\
\hline NDC 4 & $\mathrm{AD}$ (bw) & & 1 & 1 & nane & ND & $\mathrm{ND}$ & $\mathrm{ND}$ & $\mathrm{ND}$ & 0 & $\mathrm{ND}$ & $\mathrm{m}$ & 82 \\
\hline
\end{tabular}

List of human brain samples used in this study. Pathological diagnosis (PathDx), Lewy body disease (LBD) classification, amyloid plaque score (Thal phase), Braak stage, Consortium to Establish a Registry for Alzhiemer's disease (CERAD) protocol, $2 \%$ SDS and 70\% Formic Acid (FA) solubilized A $\beta 40$ and 42 (13), cerebral amyloid angiopathy (CAA), apolipoprotein E (APOE) genotype, gender, and age are reported. AD, Alzheimer's disease; PA, Pathological aging; NDC, non-demented controls; OA, optic atrophy; tr, transynaptic degeneration; GBA, gross brain atrophy; $x$, below detection levels; ND, not determined, $f$, female; $m$, male.

Table 2. Mouse strains used in this study. 


\begin{tabular}{|c|c|c|c|c|c|}
\hline Mausa Line & Transqene & Age of ansat of paftology (mo) & Descripfon of paffology & CAA & Role in Study \\
\hline PrPAPPsi & APPsweind (KM $570 / 571 \mathrm{NL}, V 617 \mathrm{~F})$ & $11-14$ & dffuse and coned heurific deposits & meringesl & hostdanor \\
\hline iTau-P301L & $4 R 0 N$ bas P $301 L$ & $18-20$ & mirimal phospho-ku & NA & host \\
\hline $\mathrm{M} 20$ & wild type aSm & NA & NA & NA & host \\
\hline P.P HUAB:PS1 & human APPywe (K595MN596LL,PS1dE9 & $5-6$ & ecred plaques & merimgeal & danor \\
\hline TeLMoAG & inducibile mouse APP swe (KM595/NL596) & -13 & primarily diffuse & meringeal & danar \\
\hline 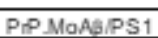 & mouse APP swe (K595MN596L).PS1dE9 & $12-13$ & alaques, mall and der & & dangr \\
\hline
\end{tabular}

Cerebral amyloid angiopathy (CAA), not applicable (NA).

\section{Figures}


a

Injection of $A D$,

\section{PA, or NDC}

To

APPsi

iTau-P301L

APPsi/iTau-P301L

b
NTg

.

PrP.APPsi/Tau-P301L, 12mo

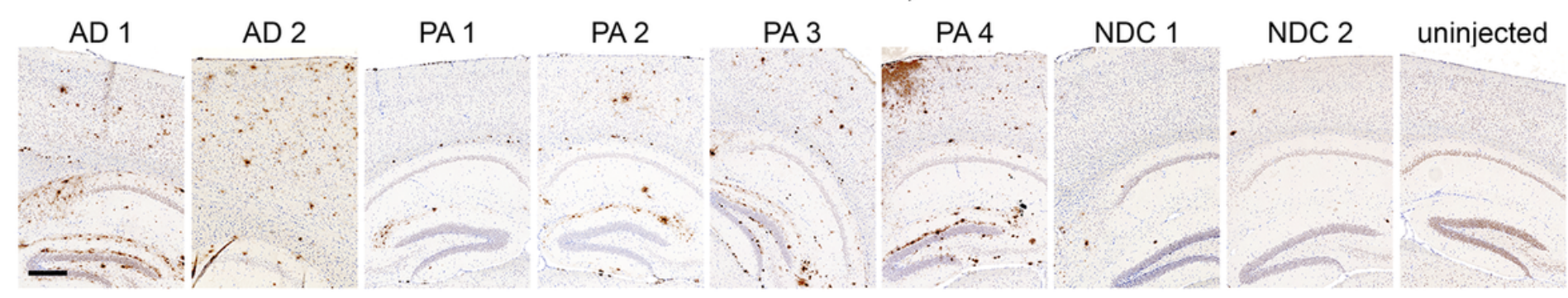

C

PrP.APPsi, 12mo

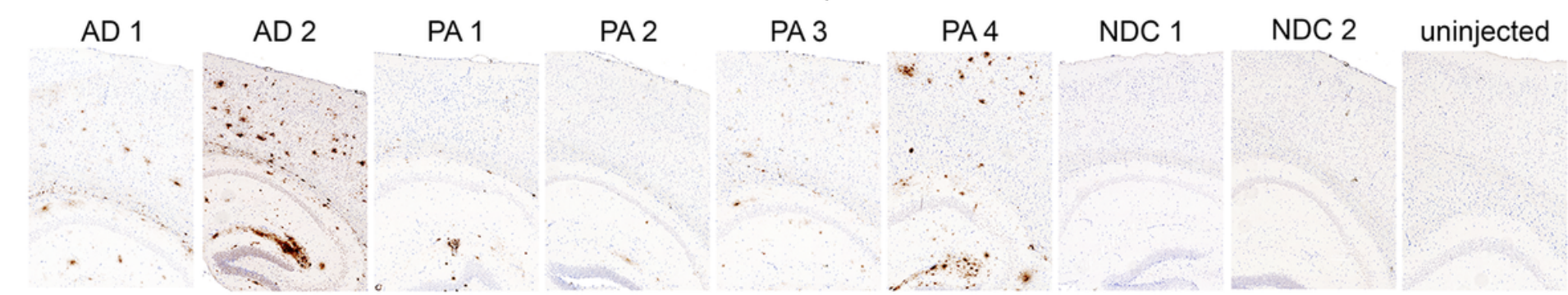

d

PrP.APPsi/Tau-P301L

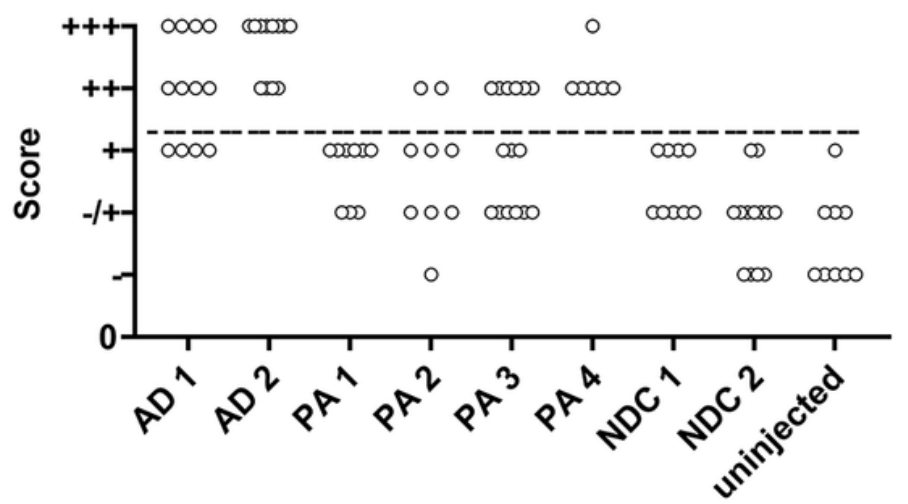

$12 \mathrm{mo}$

Pathology

ELISA

Behavior
$18 \mathrm{mo}$

Pathology

Pathology

e

PrP.APPsi

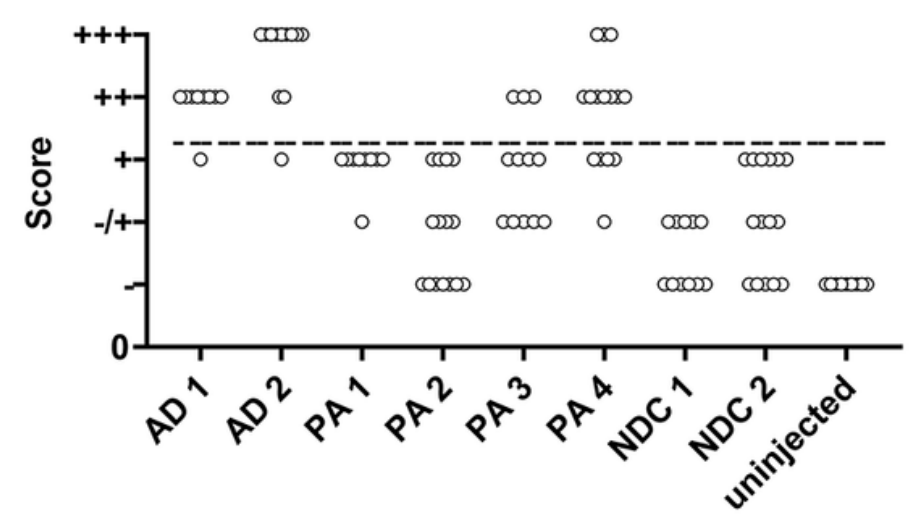

Figure 1

Cerebral injection of brain lysate at P0 induces widespread, robust amyloid deposition. Overall schematic of experiments (a) Images of PrP.APPsi/Tau-P301L (b) and PrP.APPsi (c) mice injected with AD, PA, or NDC lysate at P0. Brain sections (hemibrain) stained with biotinylated anti-A $\beta$ mAb Ab5 (anti-A $\beta$ 1-16) and counter stained with hematoxylin. Cases with the most abundant amyloid pathology are shown. Scale bar: $100 \mu \mathrm{m}$. Qualitative analysis of amyloid deposition in seeded PrP.APPsi/Tau-P301L (d) and PrP.APPsi (e) mice. Three observers independently scored representative slides from each seeded mouse. 
The amyloid pathology in each mouse was categorized on a scale from (-) as no pathology to (+++) as abundant pathology. The number of seeded mice for each scale of amyloid burden by the three observers is reported. A subset of mice injected with NDC lysate were rated as + based on 3-5 plaques per section, and thus we set this rating as baseline (marked by a dash line). Only the two AD cases and PA 4 consistently scored above + . The number of PrP.APPsi/Tau-P301L or PrP.APPsi mice injected with each lysate, respectively, were as follows: $A D$ 1: $n=4, n=3 ; A D$ 2: $n=4, n=3 ; P A ~ 1: n=3, n=3 ; P A ~ 2: ~ n=3, n=5$; PA 3: $n=5, n=4 ;$ PA 4: $n=2, n=5 ;$ NDC 1: $n=3, n=3 ; N D C 2: n=5, n=5 ;$ uninjected: $n=3, n=3$.

a

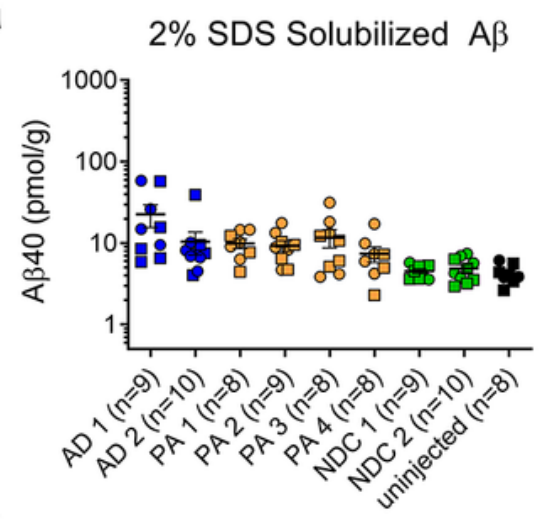

C
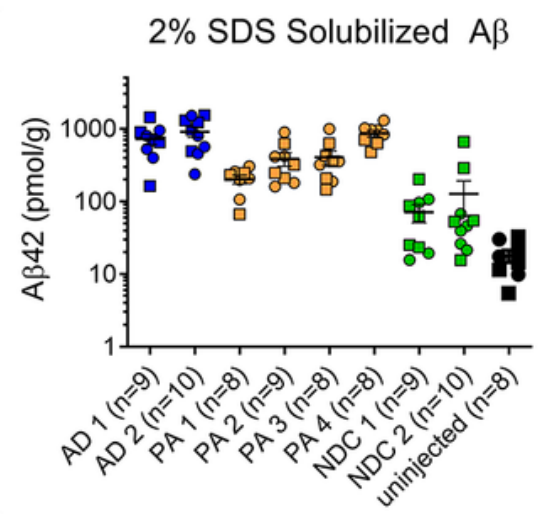

e

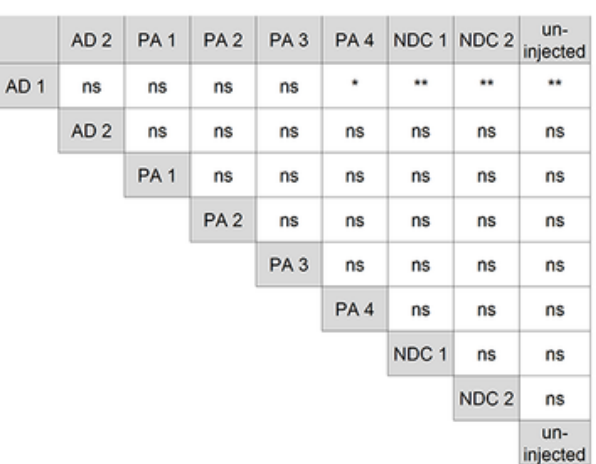

g

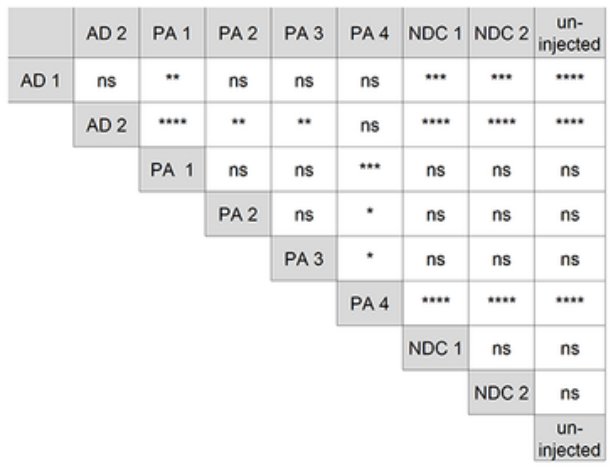

b

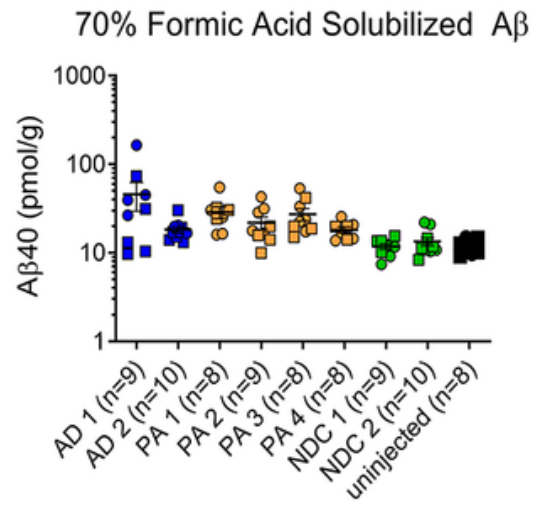

d

$70 \%$ Formic Acid Solubilized $A \beta$

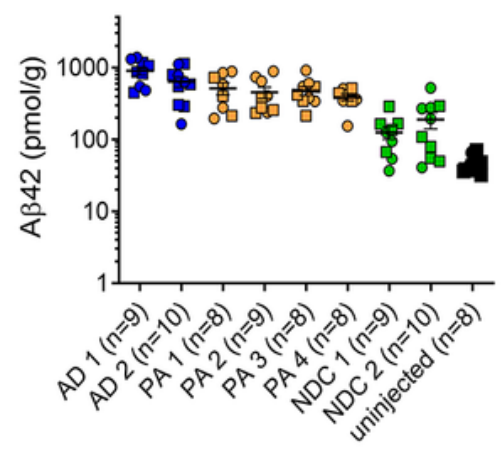

f

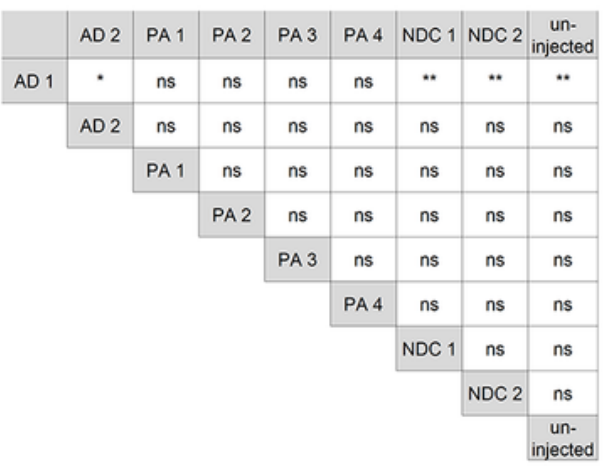

h

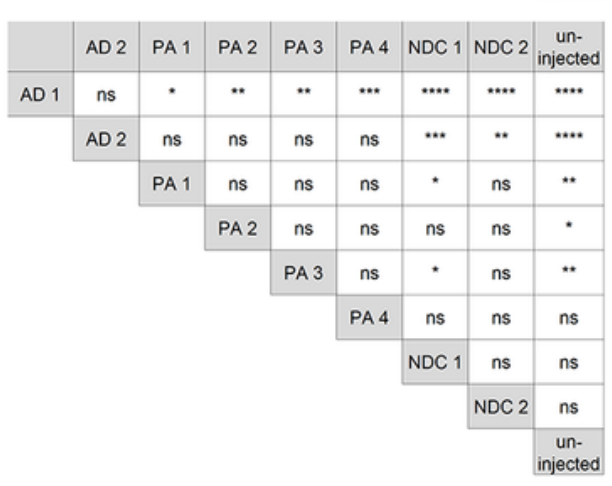




\section{Figure 2}

Biochemical analysis of sequentially extracted $A \beta 42$ and $A \beta 40$ levels by end-specific sandwich ELISA. PrP.APPsi/Tau-P301L and PrP.APPsi mice were seeded by AD, PA, or NDC lysate at P0 and aged 12

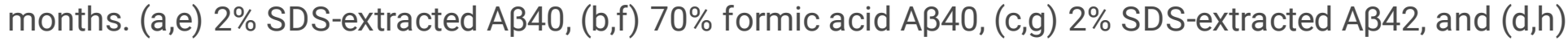
$70 \%$ formic acid AB42. Data plotted as scatter dot plot of PrP.APPsi/Tau-P301L (square) and PrP.APPsi (circle) \pm standard error of the mean. The number of PrP.APPsi/Tau-P301L or PrP.APPsi mice injected with each lysate, respectively, were as follows: $A D$ 1: $n=5, n=4 ; A D$ 2: $n=6, n=5 ;$ PA 1: $n=5, n=4 ;$ PA 2: $n=4, n=5$; PA 3: $n=5, n=4 ;$ PA 4: $n=3, n=5 ;$ NDC 1: $n=5, n=4 ; N D C$ 2: $n=5, n=5$; uninjected: $n=5, n=3$. A $\beta 42$ and $A \beta 40$ levels were quantified with corresponding one-way ANOVA with multiple comparisons test (ns, $P>0.05$; * $\mathrm{P} \leq 0.05 ; * \star, \mathrm{P} \leq 0.01 ; * \star *, \mathrm{P} \leq 0.001 ; * \star * \star, \mathrm{P} \leq 0.0001)$.

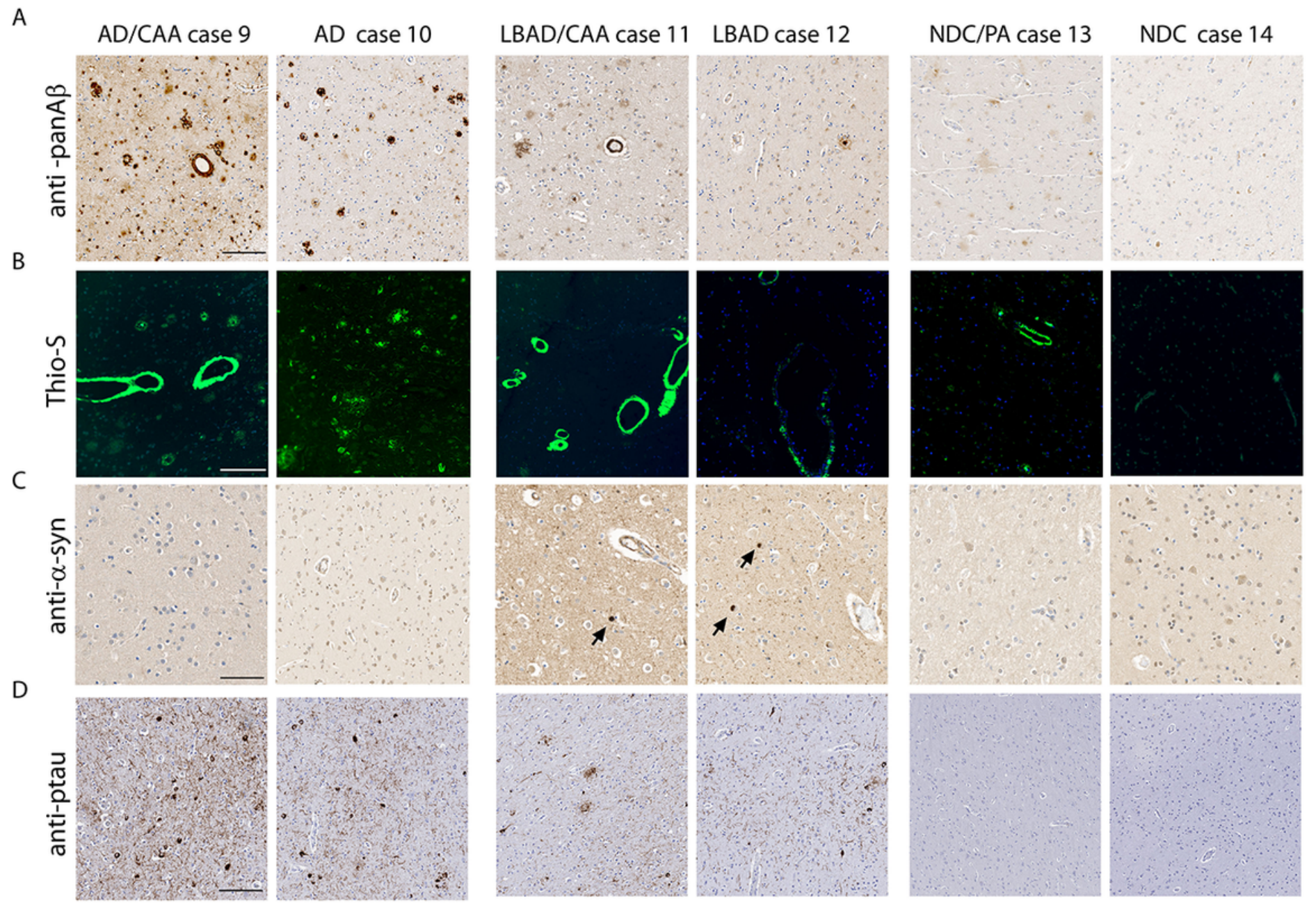

Figure 3

Characterization of $A D, A D / L B D$, and control cases. Representative image from the cortex of $2 A D$ cases, 2 AD/LBD cases, and 2 NDC cases stained with (a) biotinylated anti-A $\beta$ mAb 33.1.1 (anti-A $\beta$ 1-16). Scale 
bar: $100 \mu \mathrm{m}$, (b) Thio-S. Scale bar: $50 \mu \mathrm{m}$, (c) anti-aSyn mAb 9C10 (anti aSyn 2-21). Arrowheads depict Lewy bodies. Scale bar: $100 \mu \mathrm{m}$, and anti-ptau mAb 7F2 (pThr205). Scale bar: $150 \mu \mathrm{m}$.

a Injection of $A D$,

$A D / L B D$, or NDC

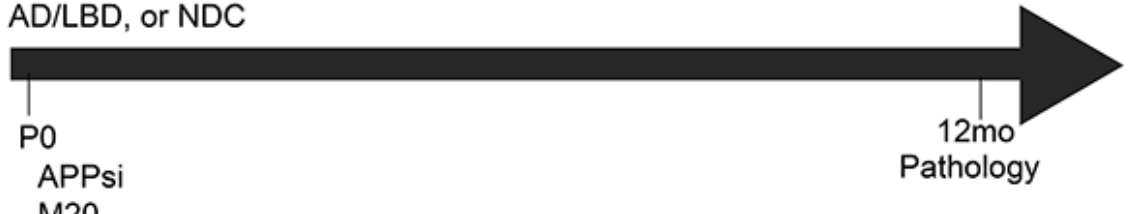

M20

APPsi/M20

NTg

b

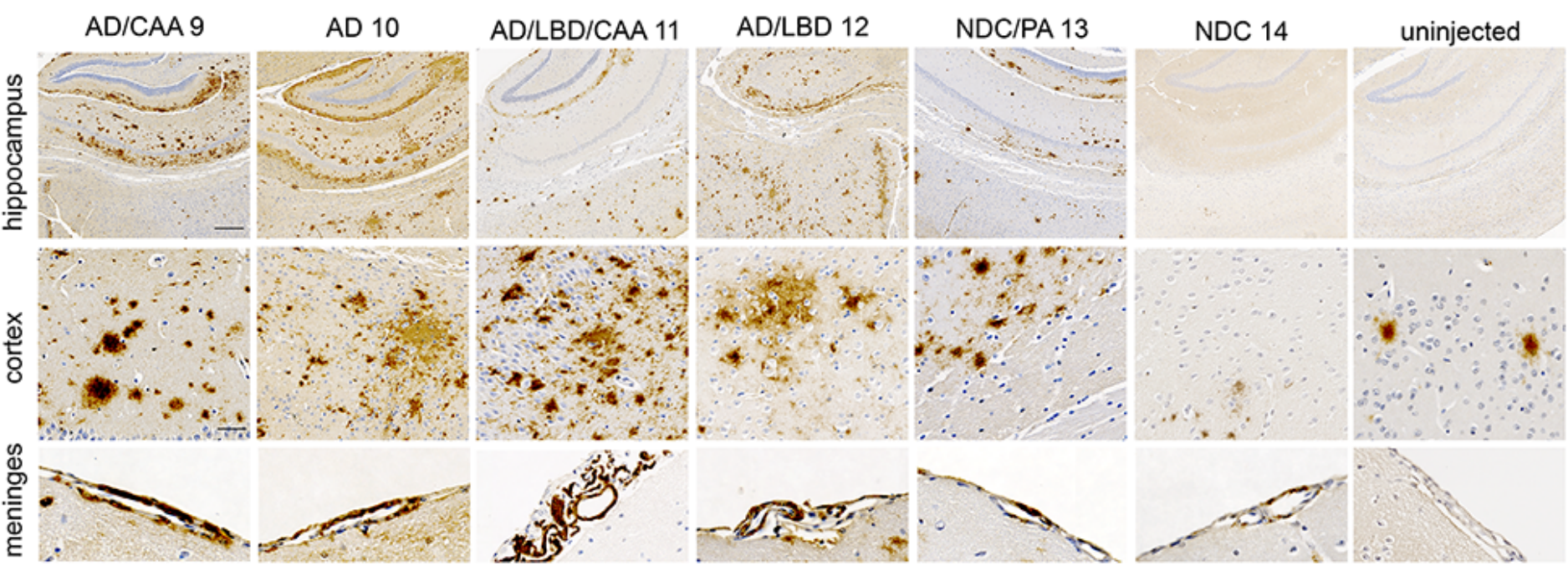

$c$
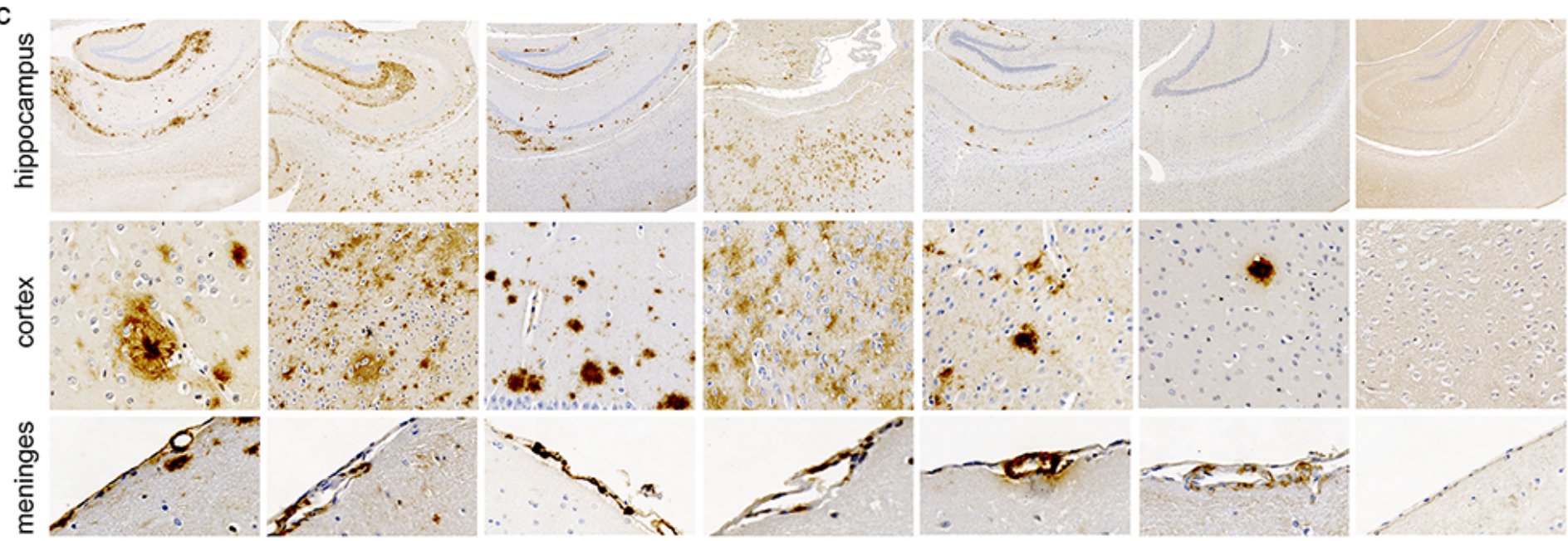

Figure 4

Cerebral injection of $A D$ and $A D / L B D$ brain lysates results in widespread amyloid deposition and CAA. (a) Newborn PO mice were injected with AD, AD/LBD, and NDC brain lysates and aged 12 months. Representative brain sections (hippocampus, cortex, and meninges) of PrP.APPsi/Line M20 and (b) PrP.APPsi mice stained with biotinylated anti-A $\beta$ mAb Ab5 (anti-A $\beta$ 1-16) and counter stained with hematoxylin. $\mathrm{n}=4-8$. Scale bar: hippocampus: $250 \mathrm{~mm}$, cortex and meninges: $50 \mathrm{~mm}$. 
a

Context

Tone


b

Context

\section{Tone}
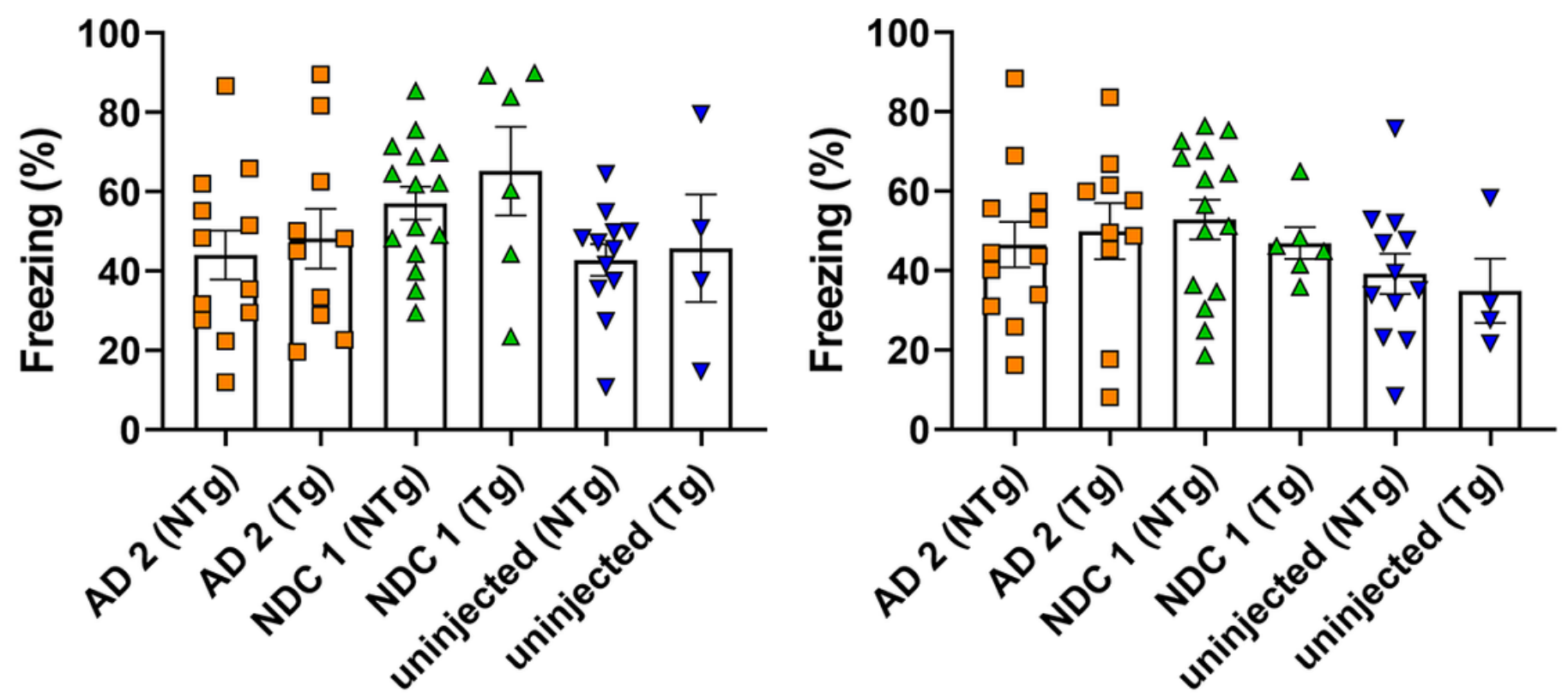

Figure 5

Diffuse amyloid seeding does not cause cognitive impairment in fear-conditioning. PrP.APPsi mice seeded by AD and NDC lysates, were aged 12 months and subjected to contextual fear conditioning. Mean percentage freezing \pm standard error of the mean exhibited by PrP.APPsi and nontransgenic (NTg) littermates injected with AD cases 1 or 2 and NDC case 7 . Uninjected mice were the control group. (a) Context and tone paradigm. (b) A subset of mice were re-tested the following week, context and tone test. $n=6-8 / \operatorname{Tg}, n=12-16 / N T g$ mice per group. 


\section{Seed Source}

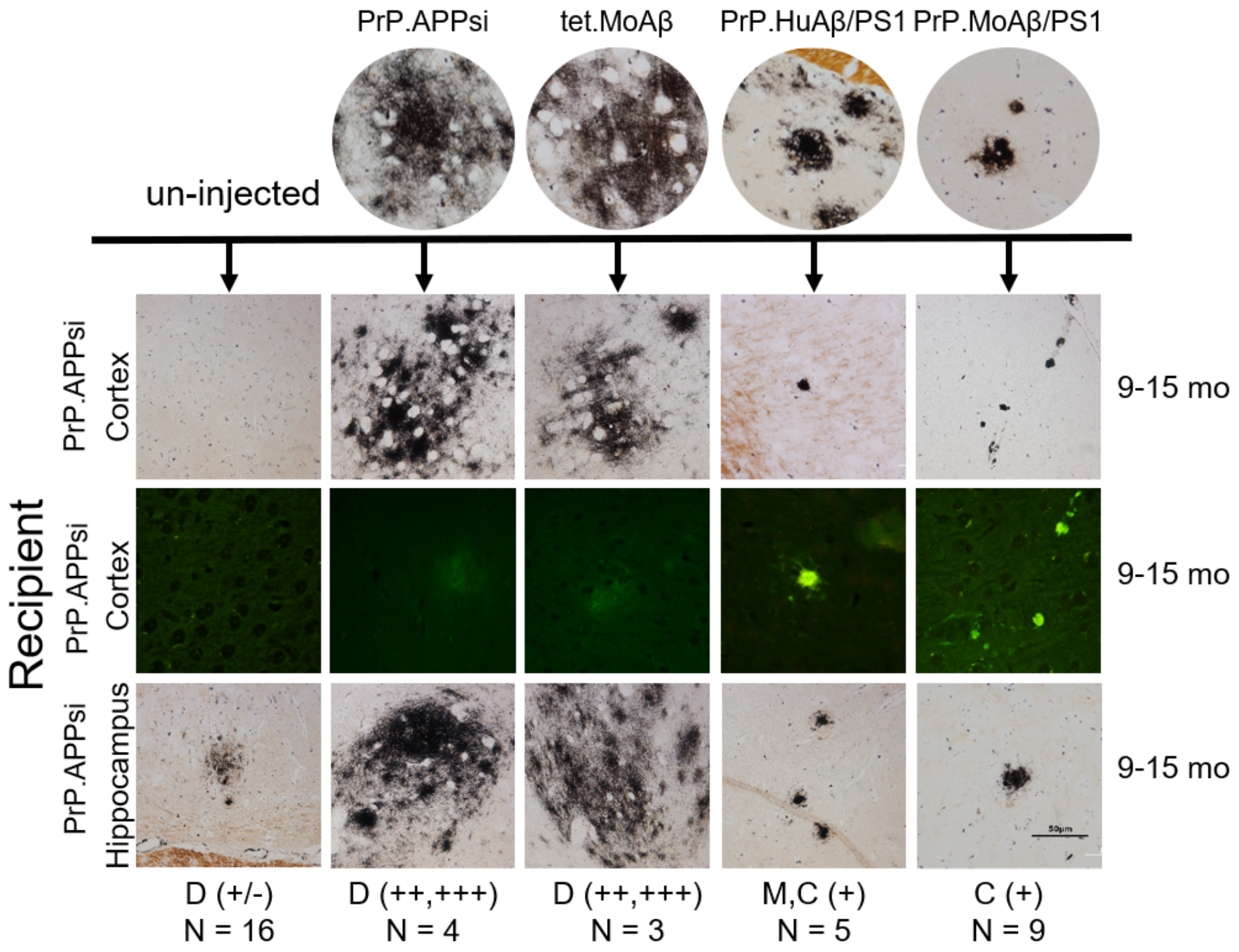

Figure 6

Comparison of seeding activity between transgenic mice that exhibit diffuse versus cored $A \beta$ pathology. We selected brains from four sources that exhibit either diffuse or cored neuritic $A \beta$ deposits (see Fig. S8). Newborn PrP.APPsi mice were injected with homogenate from each of the four sources identified at the top of the figure. Compared to uninjected mice, PrP.APPsi mice injected with brain homogenates from any source showed an induction of $A \beta$ pathology. Inoculum from aged PrP.APPsi and tet.MoA $\beta$ mice, which primarily exhibit diffuse $A \beta$ pathology $(27,38)$, robustly seeded diffuse pathology in the injected PrP.APPsi mice (severity of pathology and number of animals indicated at the bottom of the figure). Inoculum from aged PrP.HuAß/PS1 (a.k.a. APPswe/PS1dE9) and PrP.MoAß/PS1 mice, which primarily exhibit cored neuritic deposits $(27,38)$, induced the deposition of a limited number of cored $A \beta$ deposits in injected PrP.APPsi mice with little or no diffuse deposits. $D=$ diffuse $A \beta$ pathology. $M=$ mixed diffuse and cored deposits. $\mathrm{C}=$ cored, neuritic deposits. 


\section{Supplementary Files}

This is a list of supplementary files associated with this preprint. Click to download.

- Suppl.Figure1.tif

- Suppl.Figure2.tif

- SupFigure3.tif

- Suppl.Figure4.tif

- Suppl.Figure5.tif

- Suppl.Figure6.tif

- Suppl.Figure7.tif

- Suppl.Figure8.tif

- Suppl.Figure9.tif

- Suppl.Figure10.tif

- Suppl.Figure11.tif 